\title{
A MULTI-WAVELENGTH INVESTIGATION OF RCW175: AN H II REGION HARBORING SPINNING DUST EMISSION
}

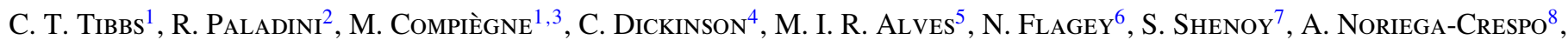 \\ S. Carey ${ }^{1}$, S. Casassus ${ }^{9}$, R. D. Davies ${ }^{4}$, R. J. Davis ${ }^{4}$, S. Molinari ${ }^{10}$, D. Elia ${ }^{10}$, M. Pestalozzi ${ }^{10}$, and E. Schisano ${ }^{10}$ \\ ${ }^{1}$ Spitzer Science Center, California Institute of Technology, Pasadena, CA 91125, USA; ctibbs@ipac.caltech.edu \\ ${ }^{2}$ NASA Herschel Science Center, California Institute of Technology, Pasadena, CA 91125, USA \\ ${ }^{3}$ Laboratoire d'Optique Atmosphérique, UMR8518, CNRS-INSU, Université Lille 1, Villeneuve d'Ascq, France \\ ${ }^{4}$ Jodrell Bank Centre for Astrophysics, School of Physics and Astronomy, The University of Manchester M13 9PL, UK \\ ${ }^{5}$ Institut d'Astrophysique Spatiale, Université Paris Sud XI, Bâtiment 121, 91405 Orsay, France \\ ${ }^{6}$ Jet Propulsion Laboratory, California Institute of Technology, Pasadena, CA 91125, USA \\ ${ }^{7}$ Space Science Division, NASA Ames Research Center, M/S 245-6, Moffett Field, CA 94035, USA \\ ${ }^{8}$ Infrared Processing and Analysis Center, California Institute of Technology, Pasadena, CA 91125, USA \\ ${ }^{9}$ Departamento de Astronomía, Universidad de Chile, Casilla 36-D, Santiago, Chile \\ ${ }^{10}$ INAF-Istituto Fisica Spazio Interplanetario, Via Fosso del Cavaliere 100, I-00133 Roma, Italy \\ Received 2012 March 18; accepted 2012 May 17; published 2012 July 12
}

\begin{abstract}
Using infrared, radio continuum, and spectral observations, we performed a detailed investigation of the $\mathrm{H}$ II region RCW175. We determined that RCW175, which actually consists of two separate H II regions, G29.1-0.7 and G29.00.6 , is located at a distance of $3.2 \pm 0.2 \mathrm{kpc}$. Based on the observations we infer that the more compact G29.0-0.6 is less evolved than G29.1-0.7 and was possibly produced as a result of the expansion of G29.1-0.7 into the surrounding interstellar medium. We compute a star formation rate for RCW175 of $(12.6 \pm 1.9) \times 10^{-5} M_{\odot} \mathrm{yr}^{-1}$, and identified six possible young stellar object candidates within its vicinity. Additionally, we estimate that RCW175 contains a total dust mass of $215 \pm 53 M_{\odot}$. RCW175 has previously been identified as a source of anomalous microwave emission (AME), an excess of emission at centimeter wavelengths often attributed to electric dipole radiation from the smallest dust grains. We find that the AME previously detected in RCW175 is not correlated with the smallest dust grains (polycyclic aromatic hydrocarbons or small carbonaceous dust grains), but rather with the exciting radiation field within the region. This is a similar result to that found in the Perseus molecular cloud, another region which harbors AME, suggesting that the radiation field may play a pivotal role in the production of this new Galactic emission mechanism. Finally, we suggest that these observations may hint at the importance of understanding the role played by the major gas ions in spinning dust models.
\end{abstract}

Key words: dust, extinction - H II regions - ISM: abundances - ISM: individual objects (RCW175, G29.1-0.7, G29.0-0.6)

Online-only material: color figures

\section{INTRODUCTION}

Dust is known to be ubiquitous in our Galaxy, playing a crucial role in the heating, cooling, and reprocessing of the interstellar medium (ISM). It is therefore no surprise that dust exists within $\mathrm{H}$ II regions, and several studies have been aimed at revealing the distribution of dust within these ionized regions (e.g., Inoue 2002; Draine 2011; Paladini et al. 2012). H II regions are the result of the formation of early-type OB stars, with their exact shape and size dependent on the surrounding environment and the balance between photoionization and recombination. They provide us with an opportunity to investigate the star formation rate (SFR) within our own Galaxy, and there has also been substantial work suggesting that $\mathrm{H}$ II regions play an important role in triggering star formation (e.g., Deharveng et al. 2005; Zavagno et al. 2006). Given the importance of $\mathrm{H}$ II regions, it is essential to fully understand the physics and dynamics of these sources.

In this analysis we focus solely on the H II region RCW 175 . RCW175, located on the Galactic plane at $l=29.07$ and $b=-0.68$, was originally identified and cataloged by Sharpless (1959) and was observed in emission with an $\mathrm{H} \alpha$ filter by Rodgers et al. (1960). RCW175 is actually just one component of the entire complex that will be discussed in this work.
As shown in Figure 1, the complex consists of two separate components: G29.1-0.7, a diffuse component to the east that is visible in $\mathrm{H} \alpha$ (this is the component detected by Sharpless 1959 and Rodgers et al. 1960) and G29.0-0.6, a more compact component to the west that is heavily obscured by dust, and as a consequence, was not detectable in $\mathrm{H} \alpha$ emission. Throughout this paper we will use RCW175 to refer to the entire complex.

RCW175 is a particularly interesting H II region, as it has been observed to be a source of anomalous microwave emission (AME; Dickinson et al. 2009). AME is observed as an excess of emission with respect to the other Galactic emission mechanisms at centimeter wavelengths, namely: thermal bremsstrahlung or free-free emission produced as a result of the interaction between the free electrons and ions in the ionized gas, synchrotron emission originating from the acceleration of relativistic electrons in the Galactic magnetic fields, and thermal dust emission arising from the vibration of large dust grains. AME is often attributed to electric dipole radiation arising from rapidly spinning small dust grains, each containing a dipole moment (Draine \& Lazarian 1998), and has been observed in other Galactic H II regions (Dickinson et al. 2007; Todorović et al. 2010) along with a number of additional Galactic environments such as molecular clouds (Watson et al. 2005; Casassus et al. 2008; Tibbs et al. 2010; Planck Collaboration 2011), dark clouds 


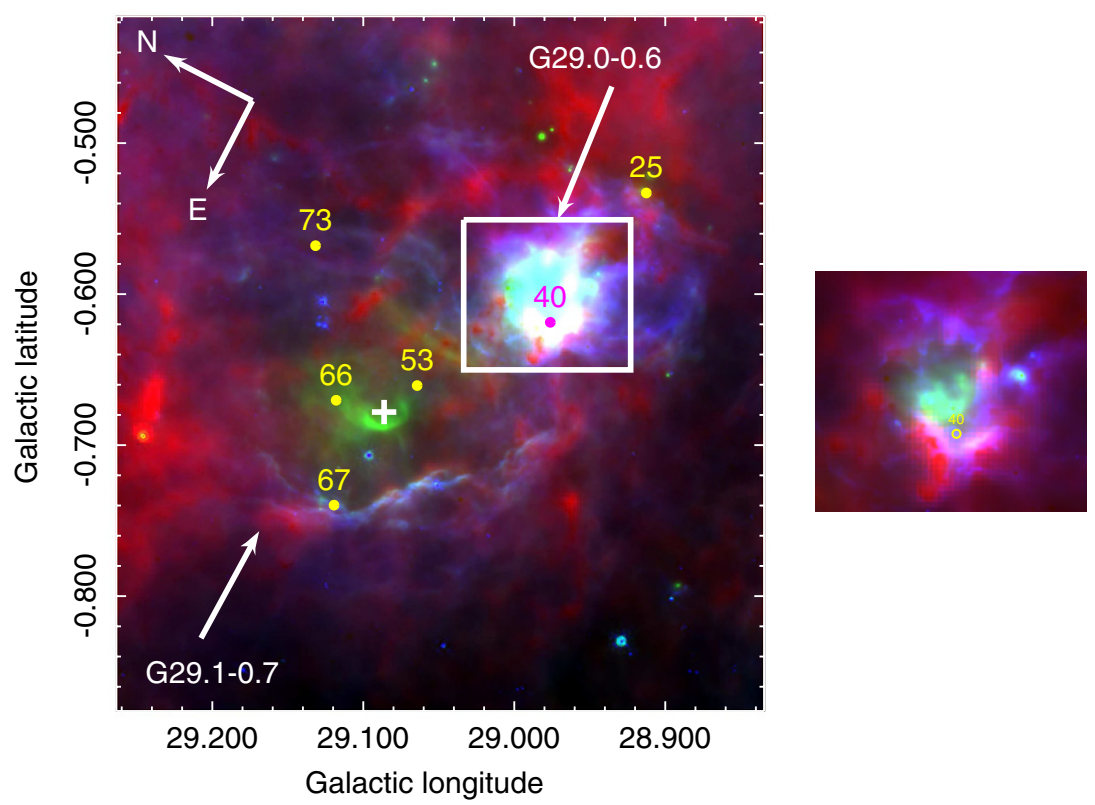

Figure 1. Three-color image of RCW175 comprising of the IRAC $8 \mu \mathrm{m}$ (blue), MIPS $24 \mu \mathrm{m}$ (green), and the SPIRE $350 \mu \mathrm{m}$ (red) maps highlighting the existing two-shell structure. The region bounded by the box is displayed separately to allow the spatial detail in this region to be seen. The $24 \mu \mathrm{m}$ emission traces the warmer dust originating from within the shells while the $8 \mu \mathrm{m}$ emission, which traces the PAH emission, is originating from the swept up dust in the shell itself. The location of the B1 II star S65-4 is marked with a cross. G29.1-0.7, the larger, diffuse component to the east, is not a complete shell, and is only bounded on one side by the swept up material. We believe that this is the result of a rupture on the north side of the H II region where the ionized gas has erupted into the surrounding ISM. G29.0-0.6, shown in the cutout, appears as more of a complete shell with pillars pointing toward the center. Also displayed is the location of the six identified YSO candidates (see Section 3.5).

(A color version of this figure is available in the online journal.)

(AMI Consortium: Scaife et al. 2009; Dickinson et al. 2010; Vidal et al. 2011), and reflection nebulae (Castellanos et al. 2011; Génova-Santos et al. 2011).

In this work, we attempt to provide a complete analysis of RCW175 in order to improve our understanding of both $\mathrm{H}_{\text {II }}$ regions in general and also the AME. The layout of the paper is as follows. Section 2 presents the data used in this work. In Section 3 we describe the morphology of the region and compute the kinematic distance, the SFR, and the dust mass. We also produce a complete spectral energy distribution (SED) of the source and discuss the presence of AME, including its possible origin and excitation mechanism within the specific environment of RCW175. Finally, in Section 4 we summarize our results and discuss our conclusions.

\section{DATA}

Performing a thorough investigation of RCW175 requires data spanning a wide range of wavelengths. However, given the angular size of RCW175 ( 25 arcmin; see Figure 1), we decided to only select data with an angular resolution $\lesssim 15$ arcmin to ensure the region was at least partially resolved. All the data used in this analysis are described in Sections 2.1, 2.2, and 2.3 corresponding to infrared (IR) data, radio continuum data, and radio spectral data, respectively.

\subsection{IR Data}

\subsubsection{IRIS Data}

The Infrared Astronomical Satellite (IRAS) data provide almost complete all-sky coverage at four bands centered at $12,25,60$, and $100 \mu \mathrm{m}$. The Improved Reprocessing of the IRAS Survey (IRIS; Miville-Deschênes \& Lagache 2005) data represent a significant improvement over the original IRAS data in regards to absolute calibration, destriping, and the removal of the zodiacal light. The IRIS data have a typical gain uncertainty of $\approx 15 \%$ and an angular resolution of $\approx 4$ arcmin. To mitigate the effect of the limited resolving power of the IRIS data, we also include higher angular resolution data from the more recent Spitzer Space Telescope and Herschel Space Observatory satellites.

\subsubsection{Spitzer Data}

To trace the warm dust in RCW175, we used mid-IR observations from the Spitzer GLIMPSE (Churchwell et al. 2009) and MIPSGAL (Carey et al. 2009) Legacy Programs. Both of these programs involved mapping the inner Galactic plane $\left(-60^{\circ}<l<60^{\circ}\right)$ and, when combined, produce a wavelength coverage of 3.6, 4.5, 5.8, 8.0, 24, and $70 \mu \mathrm{m}$. In this analysis, we decided to use only the $8 \mu \mathrm{m}$ data from the GLIMPSE survey and the $24 \mu \mathrm{m}$ data from the MIPSGAL survey. The reasons for neglecting the three shortest wavelength IRAC bands is that the point-source density increases significantly with the decrease in wavelength, and at these shorter wavelengths the spectrum can be contaminated by the presence of ionic and molecular lines, therefore making it substantially more difficult to accurately investigate extended emission. We also ignored the MIPS $70 \mu \mathrm{m}$ observations, which suffer from responsivity variations, as there are now superior quality data obtained with Herschel at the same wavelength (see Section 2.1.3).

Both the IRAC $8 \mu \mathrm{m}$ data, with an angular resolution of 2 arcsec, and the MIPS $24 \mu \mathrm{m}$ data, with an angular resolution of 6 arcsec, were reprocessed independently from the Spitzer Science Center pipeline. First, an extended emission correction was applied to the $8 \mu \mathrm{m}$ data to ensure that the IRAC calibration, which is based on point sources, was effectively applied to the extended emission. Second, both the 8 and $24 \mu \mathrm{m}$ data were 
corrected for the contribution of zodiacal light, which if not subtracted can result in an overestimate of the emission at these wavelengths by up to a factor of two. Both data sets were then subjected to source extraction, as the removal of the sources allows us to better investigate the diffuse emission. The final step in the reprocessing was to perform an overlap correction to ensure a consistent background at each wavelength. Given the data reprocessing, we assume a typical uncertainty of $10 \%$ for the extended emission in both the IRAC $8 \mu \mathrm{m}$ and the MIPS $24 \mu \mathrm{m}$ data.

\subsubsection{Herschel Data}

To complement the mid-IR Spitzer observations, data from the Herschel Space Observatory ${ }^{11}$ (Pilbratt et al. 2010), observed as part of the Hi-Gal Key Program (Molinari et al. 2010), were also incorporated into this analysis. Hi-Gal, like both GLIMPSE and MIPSGAL, is a photometric survey of the inner Galactic plane $\left(-60^{\circ}<l<60^{\circ},|b|<1^{\circ}\right)$ using both the PACS (Poglitsch et al. 2010) and SPIRE (Griffin et al. 2010) photometers at $70,160,250,350$, and $500 \mu \mathrm{m}$. These data, with an angular resolution between $6 \operatorname{arcsec}$ at $70 \mu \mathrm{m}$ and $35 \operatorname{arcsec}$ at $500 \mu \mathrm{m}$, were reduced with the ROMAGAL pipeline as described by Traficante et al. (2011). The absolute zero level was determined through the IRIS $60 \mu \mathrm{m}$ data for the PACS $70 \mu \mathrm{m}$ observations and the IRIS $100 \mu \mathrm{m}$ data for the other four bands, in a similar manner to that described in Bernard et al. (2010). We assume a $20 \%$ uncertainty for the extended emission in the PACS and SPIRE data.

\subsection{Radio Continuum Data}

\subsubsection{NVSS Data}

The National Radio Astronomy Observatory (NRAO) Very Large Array (VLA) Sky Survey (NVSS) data at $1.4 \mathrm{GHz}$ (Condon et al. 1998) represent the highest angular resolution $(0.75$ arcmin) radio map of RCW175. However, the NVSS data were observed with the VLA interferometer in D and DnC configuration, and due to a lack of short spacings they are characterized by a lack of sensitivity on scales greater than $\sim 15$ arcmin. This makes the NVSS map useful to identify highresolution radio structures present in RCW175, but it cannot be used to determine the total flux density within the region.

\subsubsection{Effelsberg Data}

Given the limitation of the NVSS data discussed above, data from both the $21 \mathrm{~cm}$ (Reich et al. 1990b) and $11 \mathrm{~cm}$ (Reich et al. 1990a) Galactic plane surveys performed with the Effelsberg $100 \mathrm{~m}$ telescope were used in this analysis. The total power level of the $21 \mathrm{~cm}(1.4 \mathrm{GHz})$ survey data $\left(-3^{\circ}<l<240^{\circ}\right.$, $|b|<4^{\circ}$ ) was obtained based on the Stockert $21 \mathrm{~cm}$ survey (Reich 1982; Reich \& Reich 1986) and they are characterized by an angular resolution of 9.4 arcmin.

Due to the data reduction techniques used to process the $11 \mathrm{~cm}$ $(2.7 \mathrm{GHz})$ survey data $\left(-2^{\circ}<l<240^{\circ},|b|<5^{\circ}\right)$, the largescale structure had to be added separately using low-resolution ( $\approx 19$ arcmin) Stockert $11 \mathrm{~cm}$ data (Reif et al. 1987). The final map has an angular resolution of 4.3 arcmin.

Based on observations of radio sources, the temperature calibration accuracy is believed to be of the order of $10 \%$ for both the 21 and $11 \mathrm{~cm}$ data.

\footnotetext{
11 Herschel is an ESA space observatory with science instruments provided by European-led Principal Investigator consortia and with important participation from NASA.
}

\subsubsection{Parkes Data}

To complement the Effelsberg data, we used the Parkes $6 \mathrm{~cm}$ $(5 \mathrm{GHz})$ data that were obtained as part of the Haynes et al. (1978) southern hemisphere Galactic plane survey $\left(-170^{\circ}<\right.$ $l<40^{\circ},|b|<2^{\circ}$ ) using the Parkes $64 \mathrm{~m}$ telescope. These data have an angular resolution of 4.1 arcmin and typical uncertainties are estimated to be $10 \%$.

\subsubsection{Nobeyama Data}

Handa et al. (1987) observed the Galactic plane $\left(-5^{\circ}<l<\right.$ $\left.56^{\circ},|b|<1.5\right)$ at $3 \mathrm{~cm}(10 \mathrm{GHz})$ using the $45 \mathrm{~m}$ telescope at the Nobeyama Radio Observatory. These data have an angular resolution of 3 arcmin and we assume a typical uncertainty of $\approx 10 \%$.

\subsubsection{Green Bank Data}

Data observed simultaneously at 3.6 and $2.1 \mathrm{~cm}$ (8.35 and 14.35 GHz) with the Green Bank 13.7 m telescope (Langston et al. 2000) as part of the Green Bank Galactic plane survey $\left(-15^{\circ}<l<255^{\circ},|b|<5^{\circ}\right)$ were also included in this analysis. These data have an angular resolution of 9.7 and 6.6 arcmin at 3.6 and $2.1 \mathrm{~cm}$, respectively, and we assume a typical uncertainty of $10 \%$.

\subsubsection{Cosmic Background Imager Data}

The Cosmic Background Imager (CBI; Taylor et al. 2011) was a 13-element interferometer that operated in the wavelength range $1.2-0.8 \mathrm{~cm}(26-36 \mathrm{GHz})$. It was with the CBI that Dickinson et al. (2009) first detected the presence of an AME component in RCW175. Although the CBI was an interferometer, the entire RCW175 complex was observed within a single CBI primary beam (28.2 arcmin) and hence any flux loss on these scales should be negligible. The CBI synthesized beam is 4.3 arcmin and we assume a conservative uncertainty of $5 \%$ for the CBI data.

\subsubsection{WMAP Data}

Wilkinson Microwave Anisotropy Probe (WMAP) 7 yr total intensity sky maps (Jarosik et al. 2011) represent all-sky observations at $1.3,0.9,0.7,0.5$, and $0.3 \mathrm{~cm}(23,33,41,61$, and $94 \mathrm{GHz}$ ) with an angular resolution of 52.8, 39.6, 30.6, 21.0, and 13.2 arcmin, respectively. However, given the angular resolution restrictions discussed previously, we use only the WMAP $94 \mathrm{GHz}$ data in this analysis. Although the WMAP data are calibrated using the CMB dipole, and hence are very accurately calibrated, due to beam errors and other systematics we assume a conservative $5 \%$ uncertainty.

\subsection{Radio Spectral Data}

To complement all of the photometric data described in Sections 2.1 and 2.2 we also utilize spectral data to help investigate the kinematics of RCW175.

\subsubsection{H I Data}

The atomic hydrogen component of the ISM is known to be widely distributed throughout the Galaxy, and therefore, even though RCW175 is an H II region, we still wish to investigate the atomic hydrogen in the vicinity of the source. H I data of RCW175 were obtained from the VLA Galactic Plane Survey (Stil et al. 2006). These observations were performed as part of an $\mathrm{HI}$ and $21 \mathrm{~cm}$ continuum emission survey covering the 
longitude range $18^{\circ}<l<67^{\circ}$, with a latitude coverage from $|b|<1.3$ to $|b|<2.3$. The short spacing information that is missing from the VLA interferometric observations is obtained by additional observations with the Green Bank Telescope. The $\mathrm{H}$ I spectral line data have an angular resolution of 1 arcmin, a spectral resolution of $1.56 \mathrm{~km} \mathrm{~s}^{-1}$, and an rms noise of $2 \mathrm{~K}$.

\subsubsection{CO Data}

$\mathrm{CO}$ data are widely regarded as the most effective tracer of molecular hydrogen. With this in mind, we incorporate both ${ }^{12} \mathrm{CO}$ and ${ }^{13} \mathrm{CO}$ observations of RCW175 in this analysis.

The ${ }^{12} \mathrm{CO}$ observations were performed as part of the Massachusetts-Stony Brook Galactic plane CO survey (Sanders et al. 1986). These observations were performed at $2.6 \mathrm{~mm}$ $(115.3 \mathrm{GHz})$ and mapped the $J=1-0$ transition in the longitude range $8^{\circ}<l<90^{\circ}$ and the latitude range $|b|<1.05$. These data have an angular resolution of $\approx 3$ arcmin, a spectral resolution of $1 \mathrm{~km} \mathrm{~s}^{-1}$, and an rms sensitivity of $\approx 0.4 \mathrm{~K}$.

The ${ }^{13} \mathrm{CO}$ data were obtained from the Galactic Ring Survey (Jackson et al. 2006). Mapping the $J=1-0$ transition $(2.7 \mathrm{~mm}$ or $110.2 \mathrm{GHz}$ ), these observations cover the longitude range $18^{\circ}<l<55^{\circ} .7$, and the latitude range $|b|<1^{\circ}$. These data have an angular resolution of 46 arcsec, a spectral resolution of $0.21 \mathrm{~km} \mathrm{~s}^{-1}$, and an rms sensitivity of $0.13 \mathrm{~K}$.

\subsubsection{Radio Recombination Line Data}

The radio recombination line (RRL) data were produced by Alves et al. (2012) by combining the three RRLs found within the $64 \mathrm{MHz}$ bandwidth of the Parkes Zone of Avoidance H I survey (Staveley-Smith et al. 1998) data. The three RRLs, $\mathrm{H} 166 \alpha, \mathrm{H} 167 \alpha$, and $\mathrm{H} 168 \alpha$, occur at a wavelength of 21.0, 21.4 , and $21.8 \mathrm{~cm}(1.42,1.40$, and $1.37 \mathrm{GHz})$, respectively. The RRL data have an angular resolution of 14.8 arcmin, a spectral resolution of $20 \mathrm{~km} \mathrm{~s}^{-1}$, and an rms sensitivity of $\approx 0.003 \mathrm{~K}$.

\section{RESULTS AND DISCUSSION}

\subsection{Morphology}

In Figure 1, we have identified the two separate components that make up RCW175. Both of these components, G29.0-0.6 and G29.1-0.7, appear shell-like: G29.1-0.7 is a larger, more diffuse component, $\approx 8$ arcmin across, while G29.0-0.6 is more compact and only $\approx 4$ arcmin across.

Based on its shape, and using 20 and $90 \mathrm{~cm}$ VLA continuum data in conjunction with Midcourse Space Experiment $21 \mu \mathrm{m}$ data, Helfand et al. (2006) tentatively classified G29.1-0.7 as a supernova remnant (SNR) candidate. However, using all the available data presented in this analysis, we identify G29.1-0.7 as an $\mathrm{H}$ II region, which is consistent with the recent analysis performed by Alves et al. (2012).

Figure 2 displays maps of RCW175 from the radio all the way to the mid-IR. Although some of the radio maps lack the resolution to completely resolve both components, G29.0-0.6 is clearly the brighter component. This is true at all wavelengths, including the IR data. The Herschel PACS and SPIRE IR emission originating from G29.1-0.7 is restricted mainly to a dust filament located along the edge of the shell visible in the IRAC $8 \mu \mathrm{m}$ map, while G29.0-0.6 appears strongly in all the Herschel bands. Although G29.0-0.6 is the dominant component, both components are visible at all the radio frequencies, indicating the presence of ionized gas in both of them.
From Figure 1 it is possible to see that the $24 \mu \mathrm{m}$ emission appears to originate within both the diffuse shell of G29.10.7 and the compact shell of G29.0-0.6, while conversely the $8 \mu \mathrm{m}$ emission largely avoids these regions, with the bulk of the $8 \mu \mathrm{m}$ emission being concentrated around the edge of the shells in filamentary structures. This concept has previously been observed in other HiI regions (e.g., Povich et al. 2007; Deharveng et al. 2010; Paladini et al. 2012), and is generally explained in terms of the classical scenario of the formation of $\mathrm{H}$ II regions: the ionized gas, produced by the ionizing $\mathrm{OB}$ star, expands into the surrounding environment due to pressure gradients. This expansion sweeps up the gas and dust creating an interface between the ionized gas and the surrounding material where photons of lower energy dominate, known as a photodissociation region (PDR; see Hollenbach \& Tielens 1997 for a complete review). In this hypothesis, the dust within the shell is warm, and thought to consist of small grains, while the smaller and less durable dust grains and large molecules, such as the polycyclic aromatic hydrocarbons (PAHs), may be destroyed by the harsh radiation field or swept up by the radiation pressure from the ionizing source. Since this scenario is consistent with what we observe in RCW175, we infer that RCW175 actually consists of two separate $\mathrm{H}$ il regions.

In addition to the two-shell structure, also visible in Figure 1 is the location of the B1 II type star, S65-4, which appears to be toward the very center of G29.1-0.7. S65-4 is part of a five star cluster identified by Forbes (1989), and is the only OB star in the Reed (2003) catalog within the vicinity of RCW175. We, therefore, believe that S65-4 is the ionization source that produced the diffuse shell, G29.1-0.7. The lack of any known OB stars associated with G29.0-0.6 does not suggest that there are none present, but instead can probably be explained by the fact that this more compact component is much more heavily obscured by dust, as can be seen by looking at the longer wavelength IR maps in Figure 2.

Having identified what we believe to be two individual H II regions within RCW175, we use the available spectral data to determine the relationship between them. By looking at the ${ }^{12} \mathrm{CO}$ and ${ }^{13} \mathrm{CO}$ spectral data displayed in Figures 3 and 4 , we determined that both G29.0-0.6 and G29.1-0.7 appear at a similar velocity of $\sim 50 \mathrm{~km} \mathrm{~s}^{-1}$. In addition to both components appearing at the same velocity, they also appear to be spatially associated with each other, with the CO emission in G29.10.7 restricted to the dust filament along the shell boundary and G29.0-0.6 being located at one end of this filament. This association implies that both components are at a similar distance and are actually interacting with each other.

In general, we find that the $\mathrm{CO}$ emission appears to spatially replicate the Herschel PACS and SPIRE data, which is not entirely unexpected as both are tracing the denser environments within RCW175. As has previously been mentioned, although the shell structure of G29.1-0.7 can be seen at $8 \mu \mathrm{m}$, the longer wavelength PACS and SPIRE emission is concentrated in a dust filament along the edge of the shell, but does not entirely enclose the shell. Assuming the $\mathrm{H}$ II region formation hypothesis, this implies that the ISM is much more dense in the direction of the filament, while in the other direction, the surrounding ISM provided less resistance to the radiation pressure and hence the swept up material formed a much lower density shell.

From Figure 5, it is possible to identify an RRL component associated with RCW175. However, due to the coarse angular resolution of the data, it is difficult to determine exactly where the emission is originating from within RCW175. The RRL data 


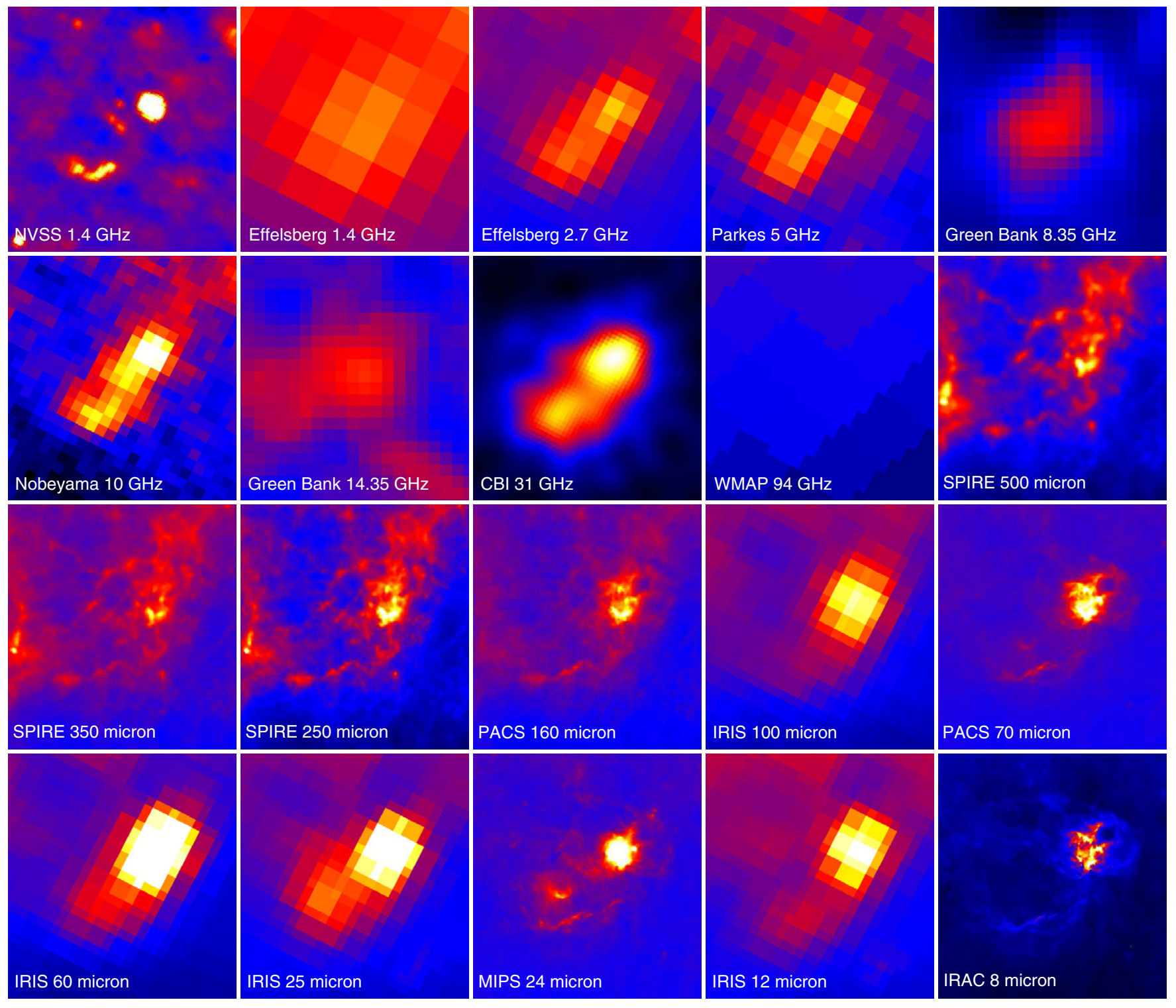

Figure 2. Maps of RCW175 all the way from the radio to the mid-IR. There are two maps at $1.4 \mathrm{GHz}$, as the NVSS map provides a high-resolution image but, due to flux loss, it cannot be used to estimate the total flux density, and so we also have the Effelsberg $1.4 \mathrm{GHz}$ map. Where the angular resolution is $\lesssim 5$ arcmin, it is possible to identify both G29.0-0.6 and G29.1-0.7. All maps are on the same coordinate system as Figure 1.

(A color version of this figure is available in the online journal.)

trace the ionized gas, therefore confirming its presence within the region. In fact, Lockman (1989) detected RRL emission from both G29.1-0.7 and G29.0-0.6, confirming that ionized gas is present in both components of RCW175, hence reinforcing the idea that both G29.1-0.7 and G29.0-0.6 are H II regions.

To further understand the morphology of RCW175, we model the IR emission using a dust emission model.

\subsection{Dust Modeling}

In the past few years, there have been substantial improvements of dust models, which have significantly increased our understanding of the ISM dust properties. One such model has been developed by Compiègne et al. (2011) and is called DustEm. ${ }^{12}$ DustEm is a sophisticated model that allows the user to parameterize various physical dust quantities to compute the corresponding emission and extinction properties. It has

\footnotetext{
12 http://www.ias.u-psud.fr/DUSTEM/
}

previously been used to characterize the dust properties in a variety of environments, such as regions of diffuse emission on the Galactic plane (Compiègne et al. 2010), the star-forming region, the Eagle nebula (Flagey et al. 2011), and the Perseus molecular cloud (Tibbs et al. 2011).

In this analysis we use DustEm in a similar manner as was used in the previous works, modeling the dust in RCW175 in terms of five dust populations: small carbonaceous dust grains, large carbonaceous dust grains, large silicate dust grains, and neutral and ionized PAHs. We merge the neutral and ionized PAHs into a single component of PAHs, use the small carbonaceous dust grains as the very small grain (VSG) dust component, and combine the large carbonaceous and silicate dust grains to form a big grain (BG) dust component. Since DustEm itself does not contain a fitting routine, we used an updated version of the SED-fitting routine developed for the analysis performed on the Perseus molecular cloud by Tibbs et al. (2011). This fitting routine is based on the IDL routine 

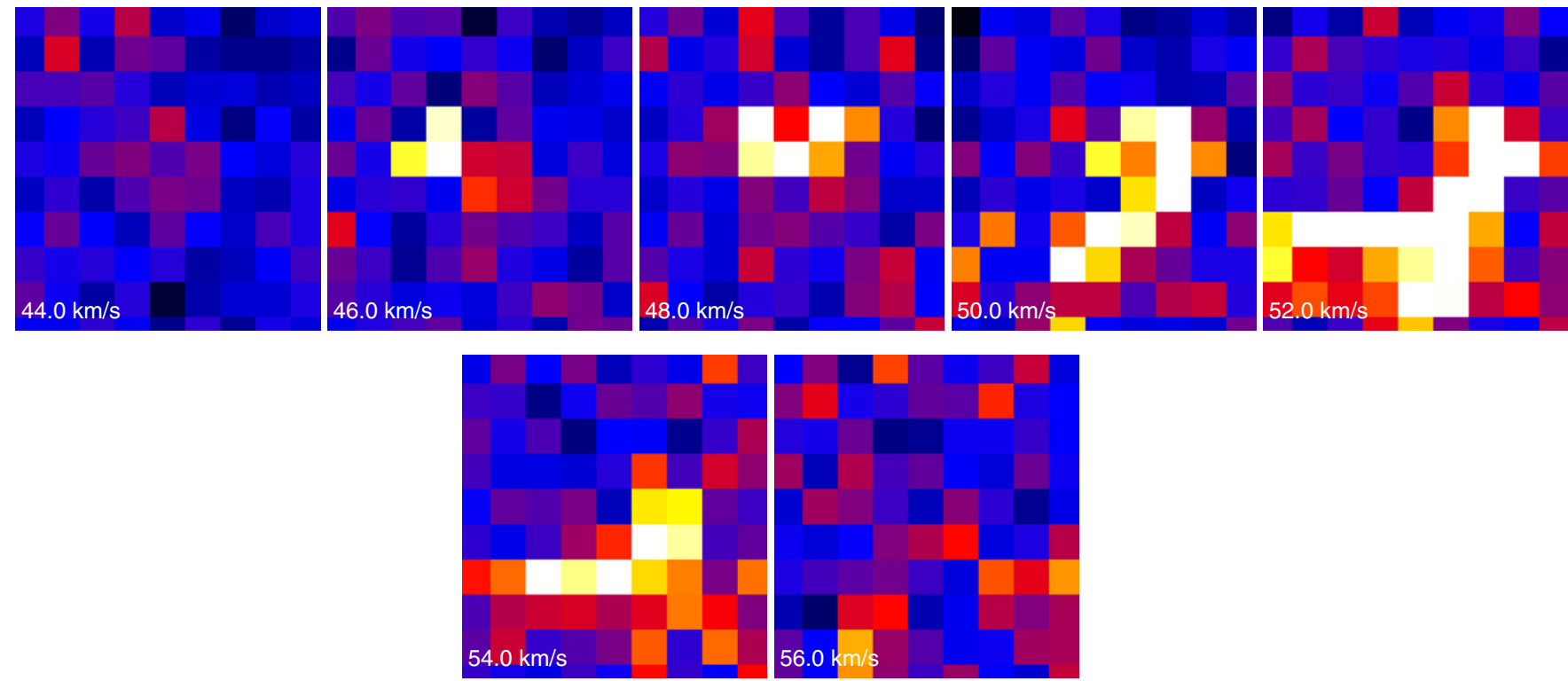

Figure 3. ${ }^{12} \mathrm{CO}$ data at seven channels across RCW175. All maps are on the same coordinate system as Figure 1.

(A color version of this figure is available in the online journal.)
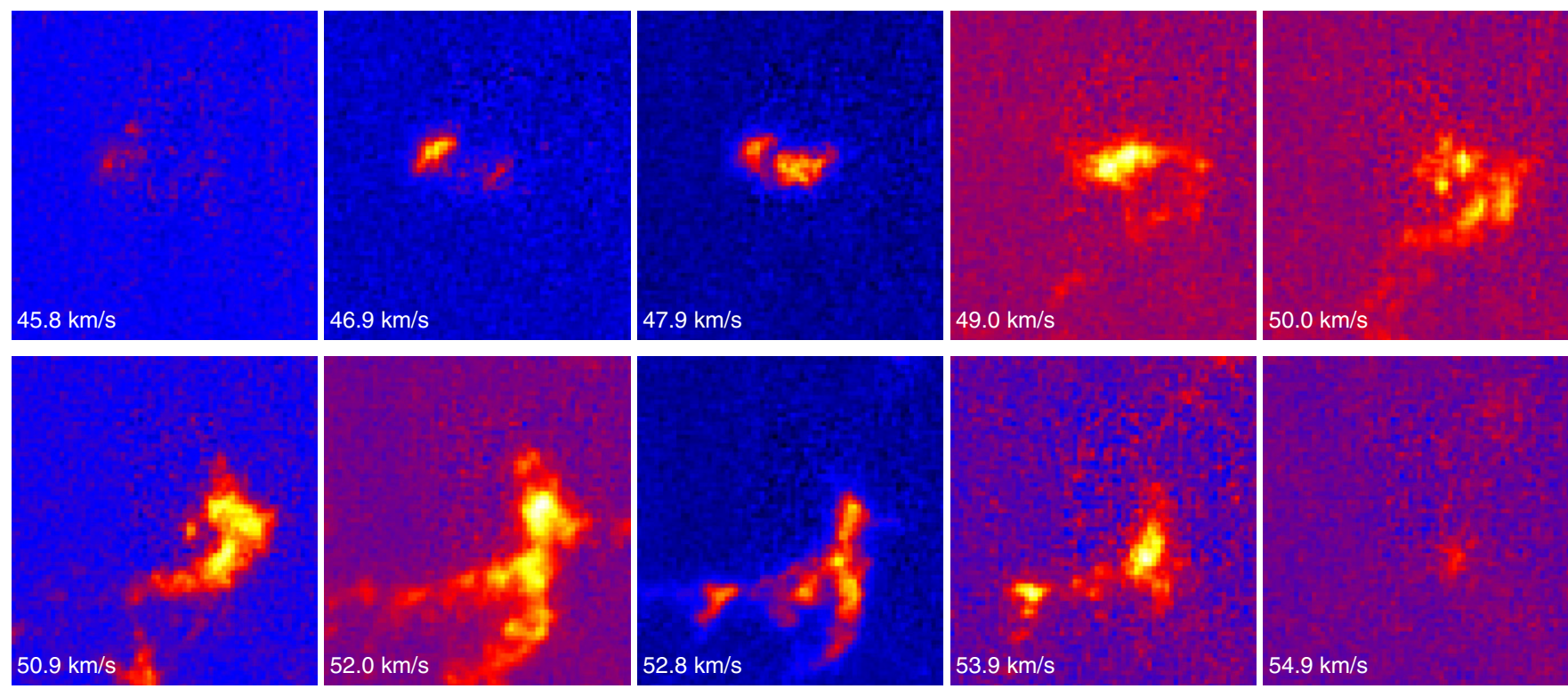

Figure 4. ${ }^{13} \mathrm{CO}$ data at 10 channels across RCW175. All maps are on the same coordinate system as Figure 1.

(A color version of this figure is available in the online journal.)
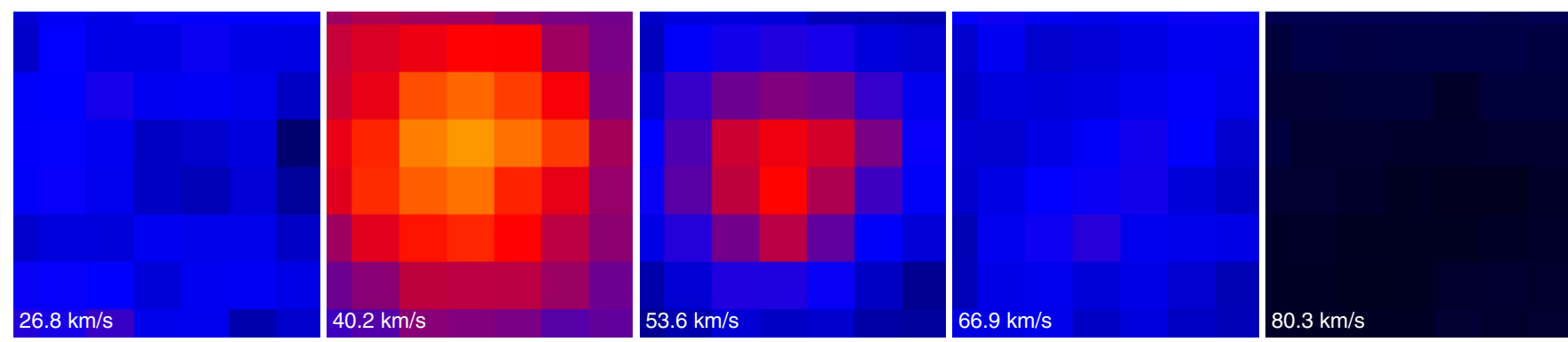

Figure 5. RRL data at five channels across RCW175. All maps are on the same coordinate system as Figure 1.

(A color version of this figure is available in the online journal.)

MPFIT (Markwardt 2009) and combines the dust emissivities computed by DustEm with the instrumental bandpass filters, deriving an estimate of the flux that would be effectively observed at a given wavelength.
For this analysis we take advantage of the new Herschel data with their superior angular resolution, and ignore the IRIS and WMAP data. Combining the Herschel data with the IRAC $8 \mu \mathrm{m}$ data and the MIPS $24 \mu \mathrm{m}$ data, results in seven bands 

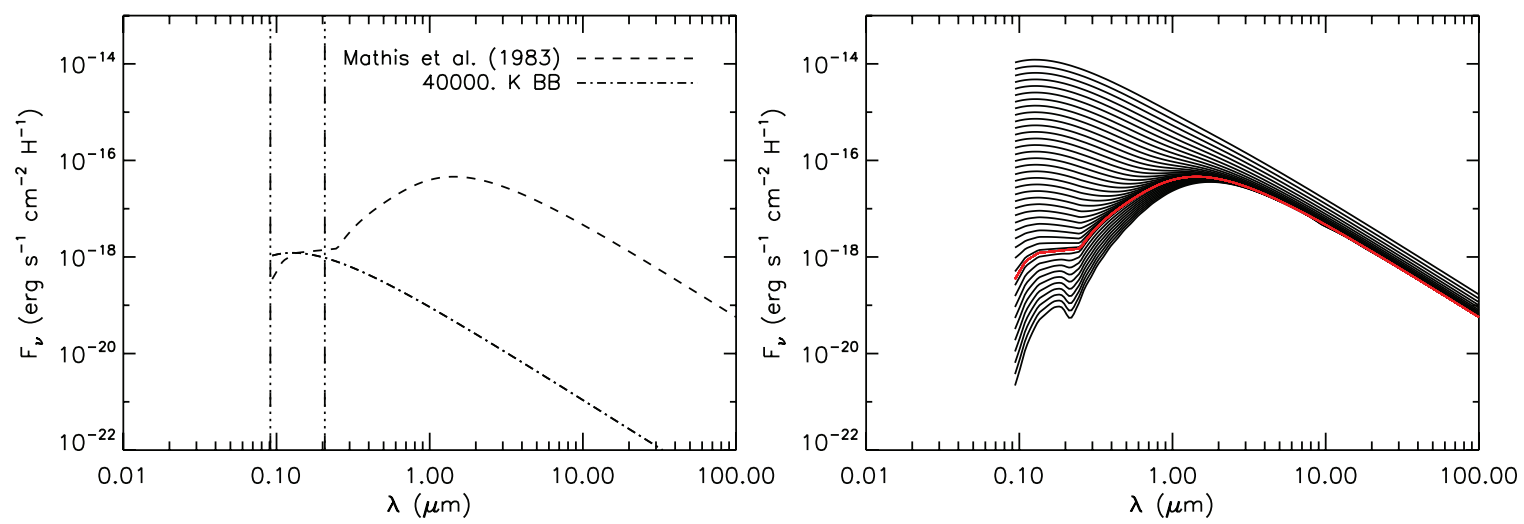

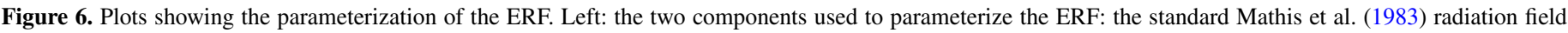

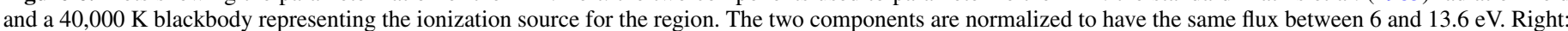

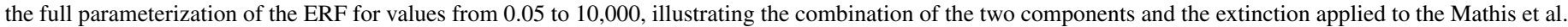
(1983) radiation field for values of $\chi_{\mathrm{ERF}}<1$. The Mathis et al. (1983) radiation field (i.e., $\chi_{\mathrm{ERF}}=1$ ) is plotted in red.

(A color version of this figure is available in the online journal.)

which provide a measure of the thermal dust emission across the entire IR spectrum. Therefore, combining DustEm with these seven IR bands allows us to characterize the three dust grain populations of interest: PAHs, VSGs, and BGs. To do this, all seven IR maps were convolved to the common angular resolution of the SPIRE $500 \mu \mathrm{m}$ map ( $35 \mathrm{arcsec}$ ) and regridded onto a unified grid of 11.5 arcsec pixels. Running DustEM with our SED-fitting routine on a pixel-by-pixel basis, we fit the seven IR data points by adjusting the abundance of the PAHs and VSGs relative to the BGs ( $Y_{\mathrm{PAH}}$ and $\left.Y_{\mathrm{VSG}}\right)$, the strength of the exciting radiation field (ERF; $\chi_{\text {ERF }}$ ), and the column density of hydrogen $\left(N_{\mathrm{H}}\right)$. In the previous work on the Perseus molecular cloud, Tibbs et al. (2011) parameterized the radiation field by simply scaling the Mathis et al. (1983) solar neighborhood radiation field, which is realistic given the lack of high-mass star formation in the cloud. However, since there is clearly a source of ionization present in RCW175, scaling the standard Mathis et al. (1983) solar neighborhood field alone would not be a good approximation. Therefore, we decided to parameterize the ERF by combining the Mathis et al. (1983) radiation field with a 40,000 K blackbody, which is the typical temperature of an OB star, representing the source of ionization. To combine these two separate components of the ERF, we normalized the $40,000 \mathrm{~K}$ blackbody to the Mathis et al. (1983) radiation field between 6 and $13.6 \mathrm{eV}$ (see Figure 6). In addition, given the presence of dust in RCW175, we implement an extinguishing of the radiation field. The final parameterization of the ERF is as follows.

1. $\chi_{\mathrm{ERF}}<1 \Rightarrow$ use the Mathis et al. (1983) radiation field extinguished by dust.

2. $\chi_{\mathrm{ERF}}=1 \Rightarrow$ use the Mathis et al. (1983) radiation field.

3. $\chi_{\mathrm{ERF}}>1 \Rightarrow$ use the Mathis et al. (1983) radiation field plus a $40,000 \mathrm{~K}$ blackbody.

In Figure 6, it is possible to see the parameterization of the ERF for the full range of values used in this analysis. Also displayed is the Mathis et al. (1983) radiation field which represents the boundary between values of $\chi_{\mathrm{ERF}}$ which are greater than, or less than, equal to 1 . Regarding our parameterization of the ERF, from Figure 6 it is apparent that the dust extinction only affects the shorter wavelengths and that for values of $\chi_{\mathrm{ERF}} \gtrsim 2500$, the blackbody becomes totally dominant.

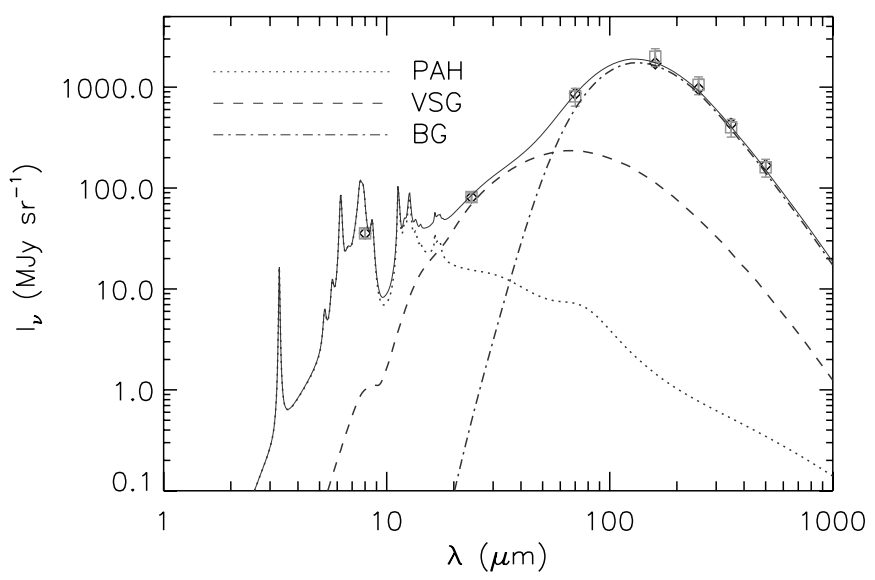

Figure 7. Spectrum of a single pixel located within the center of the shell of G29.1-0.7 showing the original IR data points (squares) and the modeled bestfitting values (diamonds). Also displayed are the three dust components required to reproduce the observed emission and the total IR emission.

The result of running our SED-fitting routine and DUSTEM on the RCW175 data was parameter maps of $Y_{\mathrm{PAH}}, Y_{\mathrm{VSG}}, \chi_{\mathrm{ERF}}$, and $N_{\mathrm{H}}$. An example of one of the fits obtained is displayed in Figure 7. This is the spectrum for a pixel within the center of the shell of G29.1-0.7 and it shows the IR data, the best-fitting values from our fitting routine and the three dust components required to reproduce the observed emission.

In addition to producing the four parameter maps, DusTEM combines the optical and thermal properties of the grains with the given ERF and calculates a temperature distribution for each size bin for each grain species. These temperature distributions are computed taking into account the multi-photon absorption processes, based on the method described by Desert et al. (1986). Therefore, to complement the parameter maps, we computed the median temperature of the $\mathrm{BG}$ size distribution, and used it as an estimate of the equilibrium temperature of the dust $\left(T_{\text {dust }}\right)$. We emphasize that it is unphysical to compute a median temperature for the other dust components as these are stochastically heated and hence are not in thermal equilibrium with the ERF. This $T_{\text {dust }}$ map, along with the four parameter maps, is displayed in Figure 8.

These maps allow us to better understand the dust properties and environment within RCW175. Looking at Figure 8, we find 

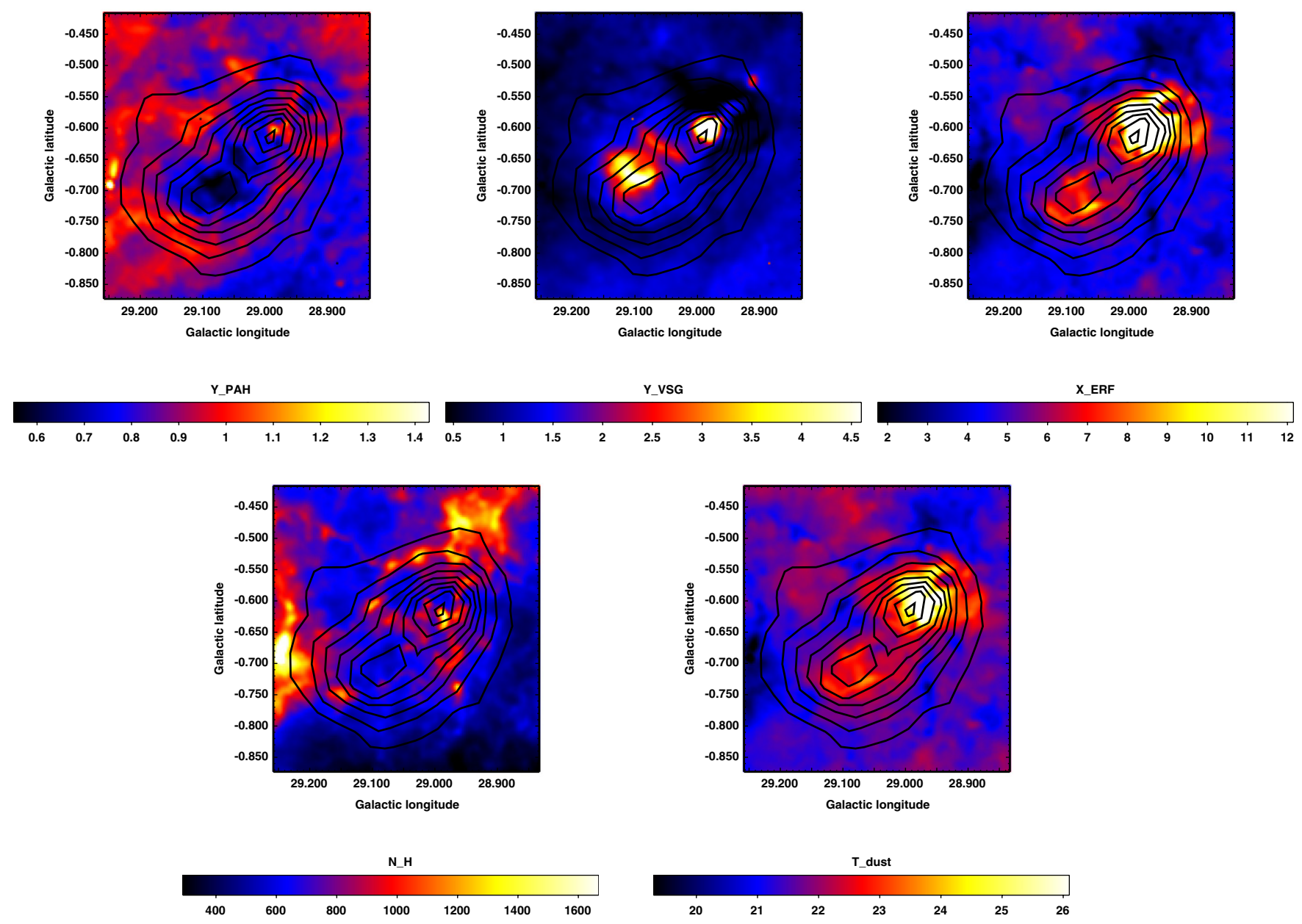

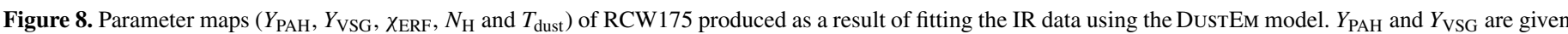

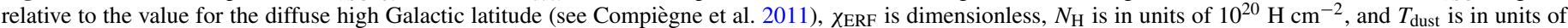

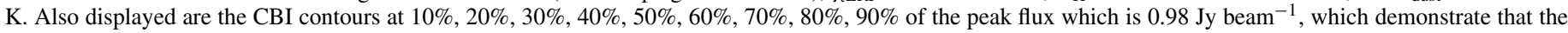
centimeter wave emission is correlated with $\chi_{\mathrm{ERF}}$ and $T_{\mathrm{dust}}$. All maps are at a common angular resolution of 35 arcsec.

(A color version of this figure is available in the online journal.)

that the abundances of the PAHs and VSGs relative to the BGs vary between $\sim 0.5-1.5$ and $\sim 0.5-10$, respectively. The maps also show that the VSGs are concentrated in the interior of both G29.1-0.7 and G29.0-0.6, while the PAHs are constrained to the edge of both components. There is also a clear deficit of PAHs within G29.1-0.7. As discussed in Section 3.1, this is consistent with the current hypothesis of $\mathrm{H}$ II region formation, with the PAHs confined to the PDR and the warmer VSGs present inside the shell.

The strength of the ERF map reveals a range of values from $\sim 2$ to 30 , with the highest values found toward G29.0-0.6, implying that there is a significant source of excitation present. This is again consistent with what we inferred from the radio and IR maps, and suggests that G29.0-0.6 is also an H II region. In G29.1-0.7, the ERF appears to be offset from the center of the shell and concentrated toward the dust filament along the edge of the shell.

The column density map reveals that the highest densities $\left(\sim 1.5 \times 10^{23} \mathrm{H} \mathrm{cm}^{-2}\right)$ are also found in the vicinity of G29.00.6 , implying that there are large quantities of dust present which we believe is the reason for a lack of observed OB stars associated with this component. Also observable is that the density is decreased toward the center of the diffuse shell of G29.1-0.7 with values of $\sim 5 \times 10^{22} \mathrm{H} \mathrm{cm}^{-2}$.
The dust temperature map closely resembles the ERF map, which is expected given the relationship between these two physical quantities, and shows that the dust temperature in RCW175 is in the range of 20-30 K, which is typical for H II regions. This map also illustrates that the highest temperatures are found toward G29.0-0.6.

Having already identified that these two H II regions are associated with each other, and based on the fact that G29.0-0.6 is more compact, has a stronger ERF, a higher dust temperature, and a higher column density, we believe that G29.0-0.6 is younger than the more evolved G29.1-0.7, and hypothesize that it may even have formed as a result of the expansion of G29.10.7 into the surrounding ISM. Such triggered star formation is ubiquitous throughout the Galaxy (e.g., Deharveng et al. 2005; Zavagno et al. 2006) essentially because the gravitational timescale of molecular gas is shorter than the typical lifetime of an O-type star. Elmegreen (1998) identified three distinct triggering mechanisms: (1) direct compression of pre-existing density enhancements in a cloud, (2) accumulation of gas into a dense filament that collapses gravitationally into dense cores ("collect and collapse"), and (3) cloud collisions. Given the environment of RCW175 and the significant differences in size, and hence age, it is unlikely that this was produced as the result of cloud collisions. Assuming that G29.1-0.7 formed first, then 
Table 1

Velocity Components, and Corresponding Kinematic Distances, for RCW175

\begin{tabular}{|c|c|c|c|c|c|}
\hline Tracer & $\begin{array}{l}\text { Velocity } \\
\left(\mathrm{km} \mathrm{s}^{-1}\right)\end{array}$ & $\begin{array}{l}D_{\text {near }} \\
(\mathrm{kpc})\end{array}$ & $\begin{array}{c}D_{\text {far }} \\
(\mathrm{kpc})\end{array}$ & $\begin{array}{c}R \\
(\mathrm{kpc})\end{array}$ & $\begin{array}{c}\text { Reference for } \\
\text { Velocity Measurement }\end{array}$ \\
\hline $\mathrm{H} \alpha$ & $49.5 \pm 5.0$ & $3.2 \pm 0.5$ & $10.8 \pm 1.5$ & $5.4 \pm 0.8$ & Crampton et al. (1978) \\
\hline${ }^{12} \mathrm{CO}$ & $52.4 \pm 1.0$ & $3.3 \pm 0.3$ & $10.7 \pm 1.1$ & $5.3 \pm 0.5$ & Blitz et al. (1982) \\
\hline${ }^{12} \mathrm{CO}$ & $51.8 \pm 1.1$ & $3.3 \pm 0.3$ & $10.7 \pm 1.1$ & $5.4 \pm 0.5$ & This work \\
\hline${ }^{13} \mathrm{CO}$ & $52.1 \pm 0.5$ & $3.3 \pm 0.3$ & $10.7 \pm 1.1$ & $5.4 \pm 0.5$ & This work \\
\hline $\mathrm{HI}_{\mathrm{I}}$ & $44.8 \pm 1.3$ & $2.9 \pm 0.3$ & $11.0 \pm 1.1$ & $5.6 \pm 0.6$ & This work \\
\hline RRL & $47.7 \pm 20.0$ & $3.1 \pm 1.3$ & $10.9 \pm 4.7$ & $5.5 \pm 2.4$ & This work \\
\hline
\end{tabular}

Notes. Both the near and far distance from the Sun, $D_{\text {near }}$ and $D_{\mathrm{far}}$, and the Galactocentric distance, $R$, are reported. References for the velocity measurements are also listed. All velocities are given with respect to the local standard of rest.

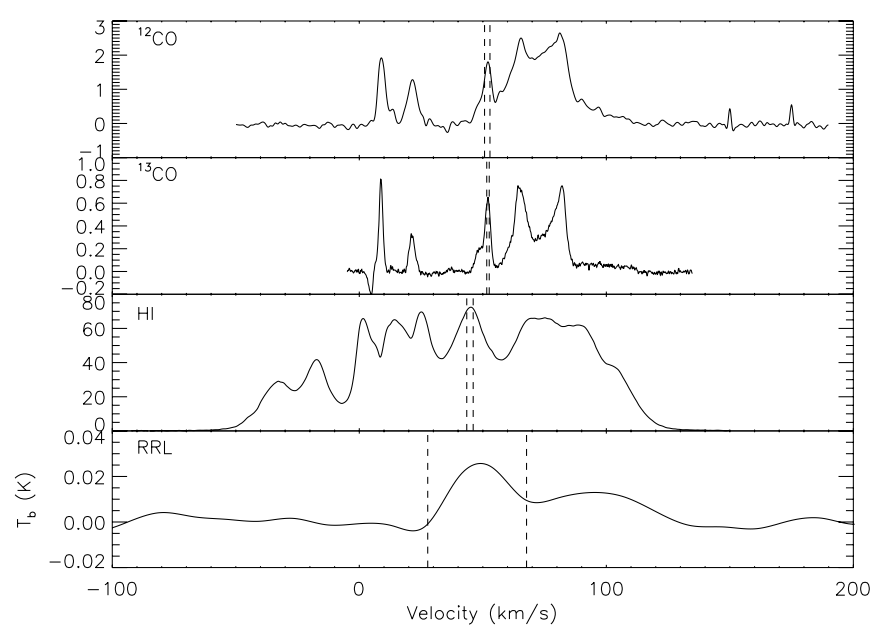

Figure 9. Velocity spectra of the ${ }^{12} \mathrm{CO},{ }^{13} \mathrm{CO}, \mathrm{HI}$, and RRL data discussed in Section 2.3. It is possible to identify the velocity components associated with RCW175 at $\sim 50 \mathrm{~km} \mathrm{~s}^{-1}$. The dashed lines indicate the $1 \sigma$ values of the corresponding velocity components listed in Table 1 .

the expansion into the surrounding ISM could have triggered the formation of G29.0-0.6 by either causing an existing density enhancement to collapse or by the collect and collapse method. It is difficult to determine which method is more favorable, however, given that G29.0-0.6 is located at the edge of the shell of G29.1-0.7, it is likely that triggering has occurred. This idea of triggered star formation will be developed further in Section 3.5.

\subsection{Kinematic Distances}

Previous estimates of the distance to RCW175 have been presented in the literature. Using the rotation curve for the northern hemisphere given by Georgelin \& Georgelin (1976) with values of $R_{0}=10 \mathrm{kpc}$ and $\Theta_{0}=250 \mathrm{~km} \mathrm{~s}^{-1}$, Crampton et al. (1978) found a kinematic distance of $3.5 \mathrm{kpc}$ to RCW175. Assuming a value of the ratio of total to selective absorption $R_{V}=3.0$, Forbes (1989) computed the reddening and distance to the ionizing B1 II star in RCW175 using the intrinsic colors given by Fitzgerald (1970) and the absolute magnitude-spectraltype calibration of Turner (1980), and found a value of $3.6 \mathrm{kpc}$.

With the spectral data described in Section 2.3 now available, it is possible to update the distance estimates to RCW175. The first step in this process is to compute the mean velocity component associated with RCW175 for each of the spectral tracers. To do this, we produced the mean spectrum within a $0.45 \times 0.45 \mathrm{deg}^{2}$ region centered on RCW175, and these spectra are displayed in Figure 9. Based on the velocity measurements in the literature and by inspecting the individual data cubes (as displayed in Figures 3-5), it is clear that the velocity component associated with RCW175 occurs around $\sim 50 \mathrm{~km} \mathrm{~s}^{-1}$. The velocity component at $\sim 50 \mathrm{~km} \mathrm{~s}^{-1}$ in each of the ${ }^{12} \mathrm{CO},{ }^{13} \mathrm{CO}$, $\mathrm{H}$ I, and RRL spectra were simultaneously fitted with a Gaussian and a linear baseline, resulting in a measure of the mean velocity component associated with RCW175 for each of the tracers. These mean velocities, along with some measurements from the literature, are listed in Table 1.

From both the spectra displayed in Figure 9 and the measurements listed in Table 1, it is possible to see that the velocities of the molecular gas tracers $\left({ }^{12} \mathrm{CO}\right.$ and $\left.{ }^{13} \mathrm{CO}\right)$ all have values which are larger than the values for the ionized gas tracers $(\mathrm{H} \alpha$ and RRL). This suggests that the molecular gas and the ionized gas may have become dissociated and that the ionized gas has ruptured and perhaps escaped the molecular gas. This idea is consistent with what we observe when we look at RCW175 in the mid- to far-IR (see Figure 2), with only one side of the shell of G29.1-0.7 being visible as a filament-like structure at the longer wavelengths. This is the same structure that is visible in the $\mathrm{CO}$ data cubes, implying that it consists of colder, denser dust that has been swept up by the radiation pressure within the center of the shell. The fact that this structure is not fully enclosing the ionized gas implies that the shell has become broken, allowing the ionized gas to leak out into the surrounding ISM. A similar conclusion can be drawn regarding the H I component, which has a lower velocity than both the molecular gas tracers and the ionized gas tracers. A lack of $\mathrm{H}_{\mathrm{I}}$ associated directly with RCW175 is not surprising since this is an H II region.

The spectra in Figure 9 also highlight the clumpy nature of the $\mathrm{CO}$ gas compared to the $\mathrm{HI}$ gas. The $\mathrm{HI}$ gas is more smoothly distributed throughout the galaxy than the $\mathrm{CO}$, and this is evidenced by the ${ }^{12} \mathrm{CO}$ and ${ }^{13} \mathrm{CO}$ spectra containing much more structure than the $\mathrm{H}$ i spectra.

To estimate the kinematic distance to RCW175, we combined the computed velocity components with a Galactic rotation curve. We used the rotation curve models of Fich et al. (1989), which holds for Galactocentric distances less than $17 \mathrm{kpc}$ making it adequate for use in this situation. We adopted the Fich et al. (1989) linear rotation curve of the form

$$
\Theta=a_{1} \Theta_{0}+a_{2} \omega_{0} R,
$$

where $\Theta$ is the rotation velocity around the Galactic center of an object at a Galactocentric distance, $R, \Theta_{0}$ and $\omega_{0}$ are the rotation velocity and angular velocity of the Sun, respectively, and $a_{1}$ and $a_{2}$ are the fitting coefficients. For the adopted values of $R_{0}=8.0 \mathrm{kpc}$ and $\Theta_{0}=220 \mathrm{~km} \mathrm{~s}^{-1}$, Fich et al. (1989) found $a_{1}=0.99334, a_{2}=0.0030385$ and $\omega_{0}=27.5 \mathrm{~km} \mathrm{~s}^{-1}$.

We note that the rotation curves of Fich et al. (1989) are based on both H I (Burton \& Gordon 1978) and CO (Blitz et al. 1982) 

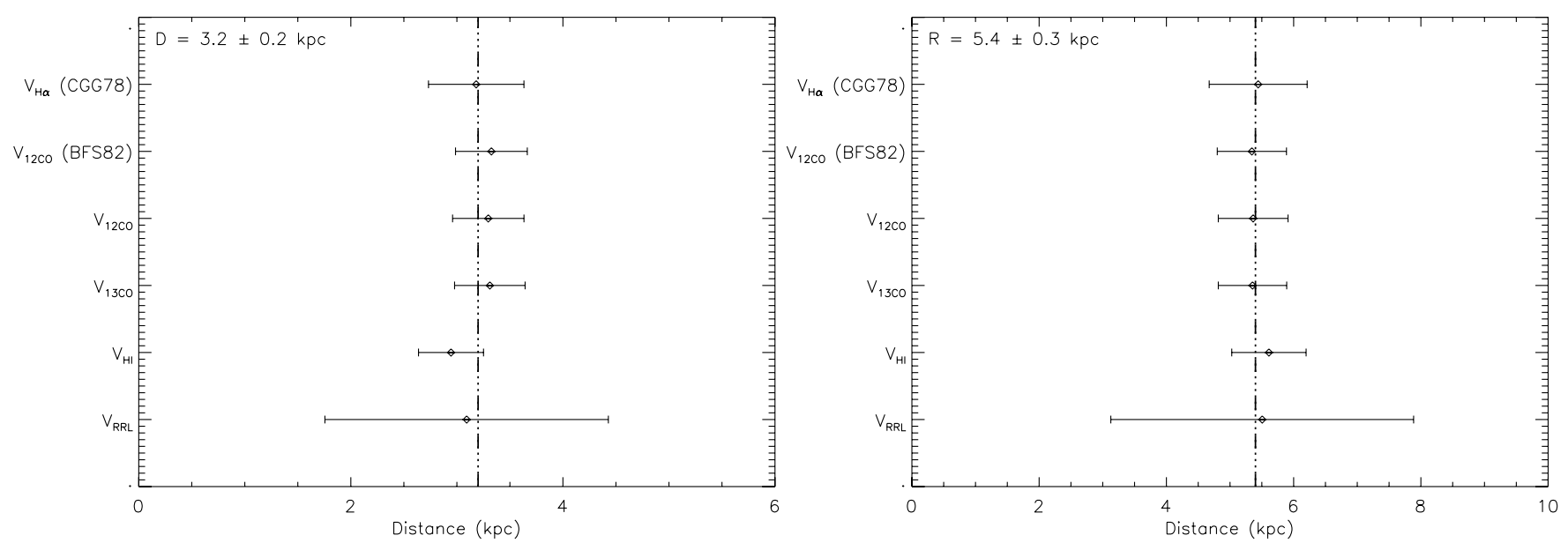

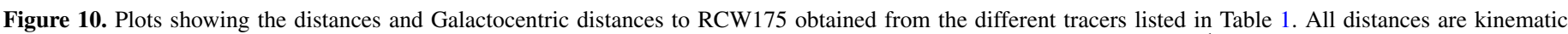
distances and have been computed using the Fich et al. (1989) rotation curve with values of $R_{0}=8.0 \mathrm{kpc}$ and $\Theta_{0}=220 \mathrm{~km} \mathrm{~s}-1$.

measurements, with 150 and 104 data points, respectively. The observations by Blitz et al. (1982) targeted all 313 objects in the Sharpless (1959) catalog and they found detections for 194 of these objects, including RCW175. This implies that RCW175 was therefore one of the data points used by Fich et al. (1989) to compute their rotation curve, using a distance of $3.5 \pm 1.2 \mathrm{kpc}$. However, given that there were a total of 254 data points, the weight of any individual region is much less than $1 \%$, and hence we feel confident in using this rotation curve to compute the kinematic distance to RCW175.

For sources with Galactic radii less than that of the Sun (i.e., sources within the solar circle) the estimated solar distance becomes degenerate as there are two solutions for every velocity, corresponding to a near distance, $D_{\text {near }}$, and a far distance, $D_{\text {far }}$. This is known as the kinematic distance ambiguity. The kinematic distances, $D_{\text {near }}$ and $D_{\text {far }}$, and Galactocentric distances, $R$, for the data discussed in Section 2.3 and for the values from the literature are tabulated in Table 1 . The distances obtained using the velocity measurements from the literature are more accurate than the corresponding previous estimates due to the updated rotation curve and updated values of $R_{0}$ and $\Theta_{0}$.

The kinematic distance ambiguity makes it difficult to determine whether $D_{\text {near }}$ or $D_{\text {far }}$ is the correct distance; however, based on the distance estimate of $3.6 \mathrm{kpc}$ to the ionizing source S65-4 located at the center of G29.1-0.7 (Forbes 1989), we select the near kinematic distance for this component. Since we have seen from the ${ }^{12} \mathrm{CO}$ and ${ }^{13} \mathrm{CO}$ data in Figures 3 and 4 , that G29.1-0.7 and G29.0-0.6 are interacting, we believe that both components are situated at the near distance.

We note that Anderson \& Bania (2009) placed G29.0-0.6 at the far distance. However, they used two separate methods to break the ambiguity, with each method giving a different result: one placing it at the near distance and the other placing it at the far distance. Given this uncertainty, we feel confident choosing the near distance for both components of RCW175. The adopted kinematic distances, $D$, and Galactocentric distances, $R$, are plotted in Figure 10. By combining all the distance estimates listed in Table 1, we compute the mean distance to RCW175, $D=3.2 \pm 0.2 \mathrm{kpc}$, and the mean Galactocentric distance, $R=$ $5.4 \pm 0.3 \mathrm{kpc}$.

\subsection{Star Formation Rate}

Various works have attempted to use the SFR from individual H II regions (e.g., Smith et al. 1978; Chomiuk \& Povich 2011) and apply them to the entire Galaxy to compute the Galactic SFR. Such an analysis requires investigating multiple $\mathrm{H}$ II regions, which is beyond the scope of this work, but computing the SFR of RCW175 will be helpful to others estimating the Galactic SFR.

Calzetti et al. (2007) developed a calibration to use monochromatic $24 \mu \mathrm{m}$ emission from extragalactic $\mathrm{H}$ II regions as an SFR indicator. Similar analyses have been performed by AlonsoHerrero et al. (2006), Pérez-González et al. (2006), and Relaño et al. (2007), each derived based on slightly different $24 \mu \mathrm{m}$ luminosity ranges. Calzetti et al. (2010) estimated an intrinsic scatter of the order of two between these different calibrations.

Recently, Chomiuk \& Povich (2011) performed an analysis investigating various SFR determinations, and in doing so they extended the Calzetti et al. (2007) extragalactic mid-IR diagnostic to the Galactic HII region M17. Following their example, we use the extragalactic calibrations to compute the SFR in RCW175.

To estimate the $24 \mu \mathrm{m}$ luminosity, we computed the flux density within RCW175 and converted this to a luminosity using

$$
L_{24 \mu \mathrm{m}}=4 \times 10^{7} \pi D^{2} S_{24 \mu \mathrm{m}} v \operatorname{erg~s}^{-1},
$$

where $D$ is the distance in $\mathrm{m}, S_{24 \mu \mathrm{m}}$ is the flux density in $\mathrm{W} \mathrm{m}{ }^{-2} \mathrm{~Hz}^{-1}$, and $v$ is the frequency in $\mathrm{Hz}$. The flux density was derived using aperture photometry in a circular aperture centered on RCW175 with a radius of $12 \mathrm{arcmin}$. An estimate of the background was obtained by computing the median value of all the pixels within an annulus, also centered on RCW175, with an inner radius of $14 \mathrm{arcmin}$ and an outer radius of $17.5 \mathrm{arcmin}$.

Given that we compute a $24 \mu \mathrm{m}$ luminosity of the order of $1 \times 10^{38} \mathrm{erg} \mathrm{s}^{-1}$ for RCW175, the calibrations established by Pérez-González et al. (2006) and Relaño et al. (2007) are the most applicable. Therefore, using the calibration of Relaño et al. (2007)

$$
\mathrm{SFR}_{24 \mu \mathrm{m}}=5.66 \times 10^{-36} L_{24 \mu \mathrm{m}}^{0.826} M_{\odot} \mathrm{yr}^{-1},
$$

where $L_{24 \mu \mathrm{m}}$ is the $24 \mu \mathrm{m}$ luminosity in units of $\mathrm{erg} \mathrm{s}^{-1}$, results in an SFR of $(12.6 \pm 1.9) \times 10^{-5} M_{\odot} \mathrm{yr}^{-1}$. This result is consistent with the SFR computed using the Pérez-González et al. (2006) calibration, which is $(12.6 \pm 1.8) \times 10^{-5} M_{\odot} \mathrm{yr}^{-1}$.

Chomiuk \& Povich (2011) explored other methods of SFR determination, including the thermal radio continuum diagnostic. Unlike the $24 \mu \mathrm{m}$ diagnostic, which uses dust emission as an 
Table 2

YSO Candidate Selection Criteria Adopted from Rebull et al. (2010)

\begin{tabular}{lcc}
\hline \hline Magnitudes & YSO Selection & Number of Sources \\
\hline 24 & {$[24]<7$} & 65 \\
$K_{S} / 24$ & $K_{s}<14$ AND $K_{s}-[24]>1$ & 58 \\
$8 / 24$ & {$[8]-[24]>0.5$ AND } & 60 \\
& $(([8]-[24]<4$ and $[8]<10)$ or $([8]-[24] \geqslant 4$ and $[8]<2.5 \times([8]-[24])))$ & 54 \\
$4.5 / 8$ & {$[4.5]<6$ AND $([4.5] \geqslant 6$ and $[4.5] \leqslant 11.5$ and $[4.5]-[8]>0.4)$} & 12 \\
$3.6 / 4.5 / 5.8 / 8$ & OR $([4.5]>11.5$ and $[4.5]<0.6944 \times([4.5]-[8])+11.22)$ & \\
& {$[3.6]-[4.5]>0.15$ and $[5.8]-[8]>0.3$ and $[3.6]<13.5$} &
\end{tabular}

indirect measure of the Lyman continuum photon flux, $N_{c}$, this method is based on the ionized gas. The Lyman continuum photon flux is the driving force of $\mathrm{H}$ II regions and the ionized gas can be observed at frequencies of a few $\mathrm{GHz}$, due to the emission of thermal bremsstrahlung or free-free radiation (as can be seen in Figure 2). Chomiuk \& Povich (2011) adopted the following relationship between the SFR and the Lyman continuum rate,

$$
\mathrm{SFR}=7.5 \times 10^{-54} N_{c} M_{\odot} \mathrm{yr}^{-1} .
$$

Therefore to compute the SFR based on the thermal radio emission, we combined this relationship with the expression for $N_{c}$ derived by Mezger et al. (1974)

$$
\begin{aligned}
N_{c}= & 4.761 \times 10^{48} a\left(v, T_{e}\right)^{-1}\left(\frac{v}{\mathrm{GHz}}\right)^{0.1} \\
& \times\left(\frac{T_{e}}{\mathrm{~K}}\right)^{-0.45}\left(\frac{S_{v}}{\mathrm{Jy}}\right)\left(\frac{D}{\mathrm{kpc}}\right)^{2} \text { photons s }^{-1},
\end{aligned}
$$

where

$$
\begin{aligned}
a\left(v, T_{e}\right)= & 0.366\left(\frac{v}{\mathrm{GHz}}\right)^{0.1}\left(\frac{T_{e}}{\mathrm{~K}}\right)^{-0.15} \\
& \times\left\{\ln \left[4.995 \times 10^{-2}\left(\frac{v}{\mathrm{GHz}}\right)^{-1}\right]+1.5 \ln \left(\frac{T_{e}}{\mathrm{~K}}\right)\right\},
\end{aligned}
$$

as defined by Mezger \& Henderson (1967), and $T_{e}$ is the electron temperature.

Having already obtained the mean Galactocentric distance to RCW175, we used the relationship between the Galactocentric distance of $\mathrm{H}$ II regions and their electron temperature, empirically derived by Paladini et al. (2004) based on a sample of 404 $\mathrm{H}$ il regions, to compute $T_{e}$. The expression from Paladini et al. (2004) is

$$
T_{e}=(4166 \pm 124)+(314 \pm 20) R \mathrm{~K},
$$

where $R$ is in units of kpc. This results in $T_{e}=5861.6 \pm 189.5 \mathrm{~K}$. Throughout the rest of this analysis we adopt a value of $T_{e}=$ $5800 \mathrm{~K}$.

Incorporating this value of $T_{e}$ into Equation (5) and the flux density, $S_{v}$, computed by integrating the flux in the Parkes $5 \mathrm{GHz}$ map (using the same aperture as for the $24 \mu \mathrm{m}$ map) we found that $N_{c} \sim 5 \times 10^{48}$ photons $\mathrm{s}^{-1}$ which, when substituted into Equation (4), results in an SFR $\approx 3.6 \times 10^{-5} M_{\odot} \mathrm{yr}^{-1}$.

This value is of the order three to four times smaller than the SFR computed from the mid-IR luminosity indicator. This discrepancy can partially be explained in terms of the intrinsic uncertainties in the different diagnostics, which is of the order two to three, with an additional contribution arising from the fact
Table 3

YSO Candidates Identified in RCW175

\begin{tabular}{lccc}
\hline \hline Source & $\begin{array}{c}\text { R.A. } \\
(\mathrm{J} 2000)\end{array}$ & $\begin{array}{c}\text { Decl. } \\
(\mathrm{J} 2000)\end{array}$ & Comments \\
\hline 25 & $18: 45: 59.9$ & $-3: 49: 12.0$ & \\
40 & $18: 46: 25.2$ & $-3: 48: 09.1$ & \\
53 & $18: 46: 43.8$ & $-3: 44: 35.3$ & \\
66 & $18: 46: 51.8$ & $-3: 41: 59.5$ & YSO candidate (Robitaille et al. 2008) \\
67 & $18: 47: 06.8$ & $-3: 43: 49.0$ & \\
73 & $18: 46: 31.4$ & $-3: 38: 27.6$ & \\
\hline
\end{tabular}

that $N_{c}$ is an underestimate of the true Lyman continuum rate. Equation (5) only computes the Lyman continuum rate of the Lyman photons that are ionizing the gas. This is not the same as computing the Lyman continuum rate of all the Lyman photons emitted by the ionizing star, as some of the Lyman photons are absorbed by dust before having the opportunity to ionize the gas. In light of this effect, we believe that the SFR of RCW175 is most accurately computed based on the $24 \mu \mathrm{m}$ emission.

\subsection{Young Stellar Objects}

As discussed by Deharveng et al. (2005), and briefly in Section 3.2, H II regions are known to play an important role in triggering star formation. Having concluded that we believe that G29.0-0.6 was possibly triggered by the expansion of G29.1-0.7 into the surrounding ISM, we would like to investigate if there is any ongoing triggering occurring within RCW175. To do this we looked at the location of candidate young stellar objects (YSOs) in relation to the general morphology of RCW175. To identify candidate YSOs we used the MIPSGAL pointsource catalog ( $\mathrm{S}$. Shenoy et al. in preparation) which contains all the MIPSGAL sources detected at $24 \mu \mathrm{m}$. Since the coverage of the MIPSGAL survey is similar to that of the GLIMPSE survey, the MIPSGAL catalog has been band-merged with the GLIMPSE sources. The GLIMPSE point-source archive contains sources at all four IRAC bands $(3.6,4.5,5.8$, and $8 \mu \mathrm{m})$ that have been band-merged with sources in all three 2MASS bands $\left(J, H\right.$, and $\left.K_{s}\right)$. The result is a band-merged source catalog with sources detected at both near- and mid-IR wavelengths that is suitable to identify YSO candidates.

Selecting only $24 \mu \mathrm{m}$ sources with a completeness and reliability of $>95 \%$, we find 95 sources within a $0.45 \times$ $0.45 \mathrm{deg}^{2}$ region centered on $\mathrm{RCW} 175$. We implemented the color-magnitude and color-color selection criteria of Rebull et al. (2010) to identify YSO candidates. These criteria are listed in Table 2 along with the corresponding number of sources in each selection, and are displayed visually in Figure 11. Combining these selection criteria resulted in a total of six YSO candidates within the vicinity of RCW175, all of which are listed in Table 3. 

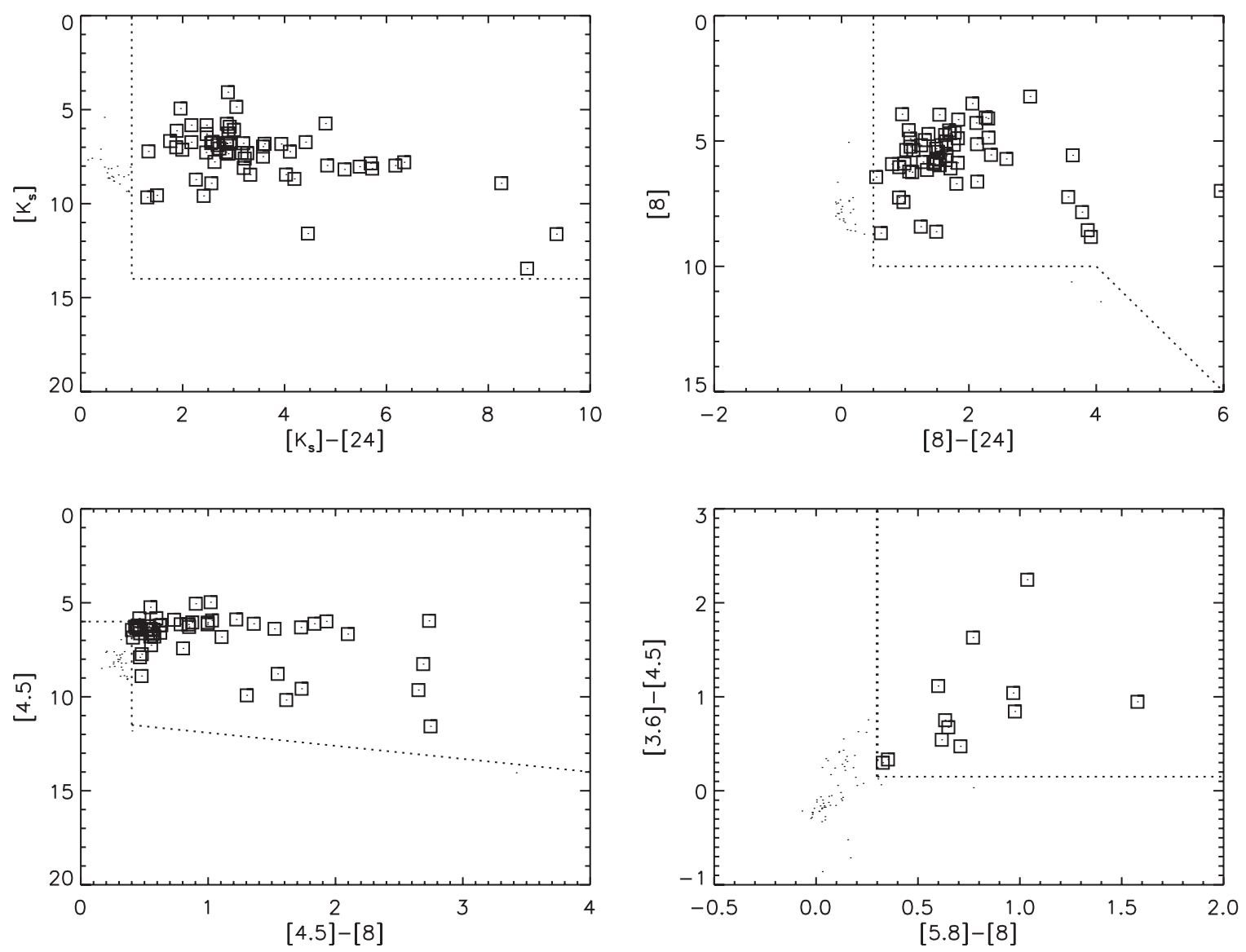

Figure 11. Color-magnitude and color-color selection criteria implemented to identify YSO candidates. All 95 sources are plotted (dots) along with the selection criteria (dotted line) and the sources that meet these criteria (squares).

A SIMBAD search within 6 arcsec (the FWHM of the $24 \mu \mathrm{m}$ point response function) of the YSO candidate position reveals that five of the sources (sources $25,40,53,67$, and 73) have no SIMBAD counterparts and one of the candidates (source 66) has previously been identified as a possible YSO candidate (Robitaille et al. 2008). Looking at the location of the six candidate YSOs displayed in Figure 1, two occur on the periphery of RCW175 (sources 25 and 73). Sources 53 and 66 are located within the diffuse shell of G29.1-0.7. The location of these sources occur at both ends of a small filament of warm dust at either side of the ionizing source S65-4. Source 67 and source 40 are found in the denser PDRs surrounding G29.10.7 and G29.0-0.6, respectively. Given the lack of any distance estimate to these sources, it is difficult to confirm that these YSO candidates are indeed associated with RCW175, however, given the location is within the dense PDR, sources 40 and 67 are likely associated with RCW175, and show that triggering may be occurring within this region.

\subsection{Dust Mass}

To estimate the total dust mass associated with RCW175, we use

$$
M_{\text {dust }}=\frac{S_{\lambda} D^{2}}{\kappa_{\lambda} B\left(\lambda, T_{\text {dust }}\right)},
$$

where $S_{\lambda}$ is the flux density, $D$ is the distance to RCW175, $\kappa_{\lambda}$ is the dust opacity, and $B\left(\lambda, T_{\text {dust }}\right)$ is the Planck function. The dust opacity was computed using

$$
2.92 \times 10^{5} \lambda_{\mu \mathrm{m}}^{-2} \mathrm{~cm}^{2} \mathrm{~g}^{-1}
$$

from Li \& Draine (2001), which is accurate for wavelengths between 20 and $700 \mu \mathrm{m}$. Since the cold BGs contribute most of the dust mass, we evaluate Equation (8) at a wavelength of $500 \mu \mathrm{m}$. Using the $500 \mu \mathrm{m}$ flux density and the equilibrium temperature of the cold dust component in RCW175 (see Section 3.7 and Table 4) along with the distance, we compute a total dust mass of $215 \pm 53 M_{\odot}$. We also computed the dust mass using the 250 and $350 \mu \mathrm{m}$ data and found that the total dust masses were in agreement (within the uncertainties) with the one computed using the $500 \mu \mathrm{m}$ data.

\subsection{Full SED}

Having discussed the various intricacies of the morphology of RCW175 in the previous sections, we now want to look at the energy budget of the region. To do this we computed the flux density of the entire complex from the radio to the mid-IR, using the same method as was used to compute the $24 \mu \mathrm{m}$ flux density described in Section 3.4.

Unlike the dust modeling analysis performed with DUSTEM in Section 3.2, we are now looking at the entire region and so no longer restrict ourselves to using only the high-resolution Spitzer and Herschel IR data. Therefore, we also incorporate the IRIS and WMAP data, as well as all the radio data discussed in Section 2.2. As stated previously, we ignore the NVSS data due to a lack of short spacings. All the flux densities and the corresponding wavelengths are listed in Table 4. Both the calibration uncertainty and the flux density uncertainty from the aperture photometry, which is computed based on the rms 


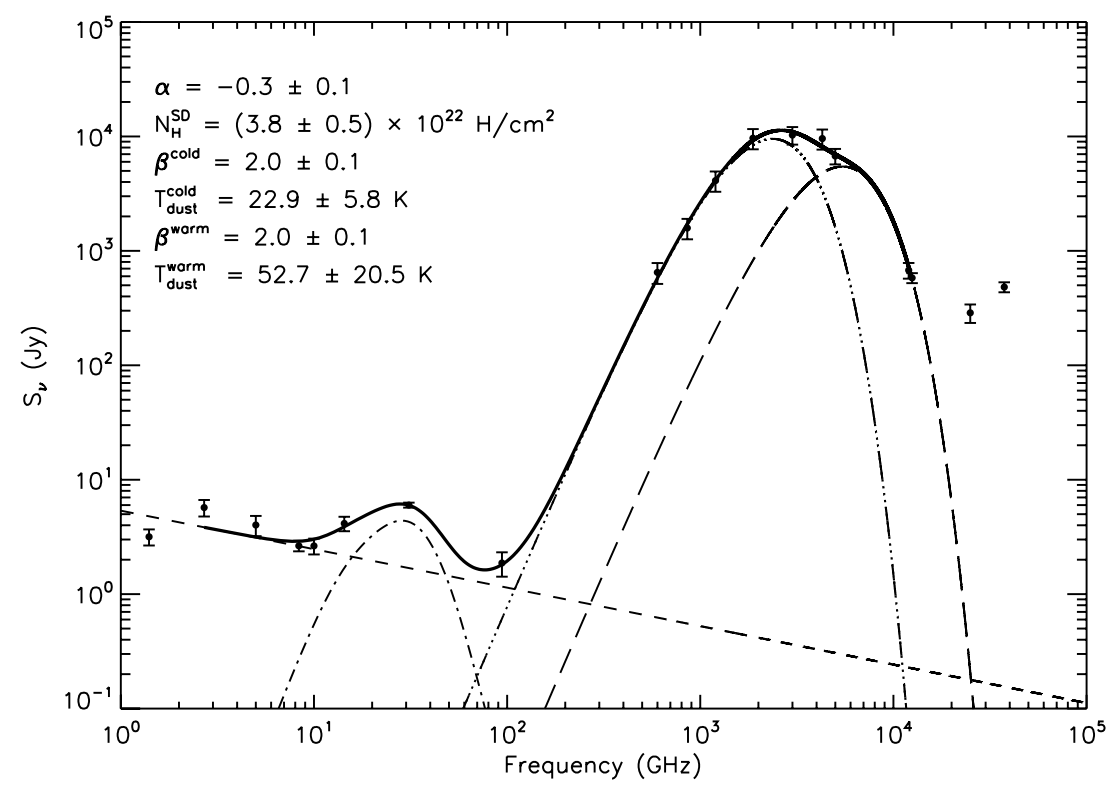

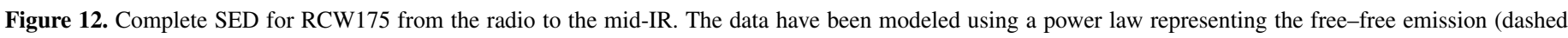

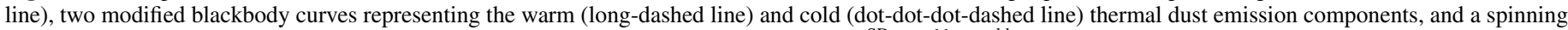

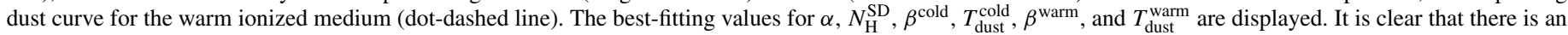
excess over both the free-free and thermal dust emission present in RCW175.

Table 4

Flux Densities for RCW175

\begin{tabular}{|c|c|c|c|c|}
\hline $\begin{array}{l}\text { Frequency } \\
(\mathrm{GHz})\end{array}$ & $\begin{array}{c}\text { Telescope/ } \\
\text { Survey }\end{array}$ & $\begin{array}{l}\text { Reference for } \\
\text { Data }\end{array}$ & $\begin{array}{l}\text { Angular Resolution } \\
\text { (arcmin) }\end{array}$ & $\begin{array}{c}S_{v} \\
(\mathrm{Jy})\end{array}$ \\
\hline 1.4 & Effelsberg $100 \mathrm{~m}$ & Reich et al. (1990b) & 9.4 & $3.2 \pm 0.5$ \\
\hline 2.7 & Effelsberg $100 \mathrm{~m}$ & Reich et al. (1990a) & 4.3 & $5.7 \pm 0.9$ \\
\hline 5 & Parkes $64 \mathrm{~m}$ & Haynes et al. (1978) & 4.1 & $4.0 \pm 0.8$ \\
\hline 8.35 & Green Bank 13.7 m & Langston et al. (2000) & 9.7 & $2.6 \pm 0.3$ \\
\hline 10 & Nobeyama 45 m & Handa et al. (1987) & 3.0 & $2.6 \pm 0.4$ \\
\hline 14.35 & Green Bank 13.7 m & Langston et al. (2000) & 6.6 & $4.1 \pm 0.6$ \\
\hline 31 & $\mathrm{CBI}$ & Dickinson et al. (2009) & 4.3 & $6.0 \pm 0.3$ \\
\hline 94 & WMAP & Jarosik et al. (2011) & 13.2 & $1.9 \pm 0.4$ \\
\hline $599.6(500 \mu \mathrm{m})$ & Herschel/SPIRE & Molinari et al. (2010) & 0.6 & $649.0 \pm 134.0$ \\
\hline $856.5(350 \mu \mathrm{m})$ & Herschel/SPIRE & Molinari et al. (2010) & 0.4 & $1580.0 \pm 322.0$ \\
\hline $1199.2(250 \mu \mathrm{m})$ & Herschel/SPIRE & Molinari et al. (2010) & 0.3 & $4110.0 \pm 829.0$ \\
\hline $1873.7(160 \mu \mathrm{m})$ & Herschel/PACS & Molinari et al. (2010) & 0.2 & $9660.0 \pm 1940.0$ \\
\hline $2997.9(100 \mu \mathrm{m})$ & IRIS & Miville-Deschênes \& Lagache (2005) & 4.3 & $10300.0 \pm 1800.0$ \\
\hline $4282.7(70 \mu \mathrm{m})$ & Herschel/PACS & Molinari et al. (2010) & 0.1 & $9580.0 \pm 1920.0$ \\
\hline $4996.5(60 \mu \mathrm{m})$ & IRIS & Miville-Deschênes \& Lagache (2005) & 4.0 & $6740.0 \pm 1050.0$ \\
\hline $11991.7(25 \mu \mathrm{m})$ & IRIS & Miville-Deschênes \& Lagache (2005) & 3.8 & $677.0 \pm 106.0$ \\
\hline $12491.4(24 \mu \mathrm{m})$ & Spitzer/MIPS & Carey et al. (2009) & 0.1 & $581.0 \pm 58.1$ \\
\hline $24982.7(12 \mu \mathrm{m})$ & IRIS & Miville-Deschênes \& Lagache (2005) & 3.8 & $287.0 \pm 52.8$ \\
\hline $37474.1(8 \mu \mathrm{m})$ & Spitzer/IRAC & Churchwell et al. (2009) & 0.03 & $483.0 \pm 48.3$ \\
\hline
\end{tabular}

of the pixel values in the background, have been incorporated into the final uncertainties of the flux densities.

The values in Table 4 are plotted in Figure 12. This SED is an updated version of the original SED presented by Dickinson et al. (2009). We have used more recent data where available (e.g., Effelsberg $1.4 \mathrm{GHz}$ ) and we have substantially improved the constraint on the Rayleigh-Jeans tail of the thermal dust distribution given the inclusion of the Herschel data. It should also be noted that even though Dickinson et al. (2009) used Gaussian fitting to compute the flux densities, while in this anlaysis we have used aperture photometry, the general consistency between both sets of data points is good.

The data between 2.7 and $12491.4 \mathrm{GHz}$, or $11 \mathrm{~cm}$ and $24 \mu \mathrm{m}$, were simultaneously fitted with a power law $\left(S_{v} \propto\right.$ $\left.v^{\alpha}\right)$, a spinning dust model, and two modified blackbody curves
$\left(S_{v} \propto v^{\beta} B\left(v, T_{\text {dust }}\right)\right)$ representing the free-free, AME, and thermal dust emission, respectively. These components will be discussed further in Sections 3.7.1-3.7.3.

\subsubsection{Low-frequency Emission}

Fitting the radio data in Figure 12 with a single power law resulted in a spectral index of, $\alpha=-0.3 \pm 0.1$. This is steeper than the typical spectral index of $\sim-0.1$ expected for optically thin free-free emission at these frequencies (Dickinson et al. 2003), however it is consistent with the value of $-0.32 \pm$ 0.15 obtained previously by Dickinson et al. (2009). To explain this steeper spectral index we believe that there must be some residual contamination from synchrotron emission in the lowfrequency radio maps which, given that synchrotron emission generally follows a power law with a spectral index of $\sim-0.7$, 
could be contributing to the steeper spectral index found in RCW175. Given that RCW175 is located on the Galactic plane, there will be a contribution from the diffuse synchrotron emission. Additionally, a search in the Green (2009) catalog of Galactic SNRs reveals three nearby $\left(<1^{\circ}\right.$ away) SNRs, G29.70.3 (Kes 75) and G28.6-0.1 both $\sim 0.7$ away, and G29.6+0.1 $\sim 0$.9 away, which may be causing synchrotron relativistic electrons to escape into the surrounding environments.

We therefore conclude that the low-frequency emission in RCW 175 is arising from the free-free emission from the ionized gas and some small contribution from background residual synchrotron emission.

\subsubsection{Thermal Dust Emission}

To represent the thermal dust emission we used a modified blackbody curve, fitting for both the dust temperature, $T_{\text {dust }}$, and the dust emissivity spectral index, $\beta$. Two curves were used to fit both the cold and warm dust components. This is necessary since we are computing the SED for the entire region, and we know that the dust within the entire region is not emitting at a single temperature. In the DUSTEM analysis, since we were fitting the SED on a pixel-by-pixel basis, we did not have this problem, and hence were able to restrict ourselves to using a single dust temperature.

The best-fitting values for the cold dust are $\beta^{\text {cold }}=2.0 \pm$ 0.1 and $T_{\text {dust }}^{\text {cold }}=22.9 \pm 5.8 \mathrm{~K}$, while for the warm dust we find best-fitting values of $\beta^{\text {warm }}=2.0 \pm 0.1$ and $T_{\text {dust }}^{\text {warm }}=52.7 \pm$ $20.5 \mathrm{~K}$. These values of the cold and warm dust components are consistent with previous estimates of dust temperatures in $\mathrm{H}$ II regions (e.g., Paladini et al. 2012). It is also noticeable that by including the Herschel data in our SED, the temperatures computed in this analysis are more accurate than the dust temperature estimated by Dickinson et al. (2009) who fitted a modified blackbody curve to the IRIS 100 and $60 \mu \mathrm{m}$ data, and obtained a temperature of $30.1 \pm 5.7 \mathrm{~K}$.

\subsubsection{Anomalous Microwave Emission}

The excess of emission observed between $\sim 10$ and $60 \mathrm{GHz}$ in the SED of RCW175 is a clear indication of the presence of AME. The peaked nature of the AME spectrum is consistent with the current theoretical models of spinning dust (Draine \& Lazarian 1998; Ali-Haïmoud et al. 2009; Hoang et al. 2010; Ysard \& Verstraete 2010; Hoang et al. 2011; Silsbee et al. 2011), and therefore we modeled the AME as a spinning dust component. To do this we used the spinning dust code, SPDUST (Ali-Haïmoud et al. 2009; Silsbee et al. 2011). For a given set of input parameters, SPDUST computes the emissivity of the spinning dust emission normalized by the hydrogen column density. We used the updated version of SPDUST, which not only accounts for dust grains spinning about their axis of greatest inertia, it also accounts for grains spinning about their nonprincipal axes.

Since we have only two data points covering the critical frequency range, we decided not to fit for the individual spinning dust parameters within SPDUST. Instead, we adopted the idealized physical parameters for the various phases of the ISM (Draine \& Lazarian 1998; warm ionized medium, warm neutral medium, cold neutral medium, molecular cloud, dark cloud, reflection nebula, and PDR) within SPDUST and fitted for the column density. This simply involves scaling the amplitude of the spinning dust curve and has no effect on its shape or peak frequency. We performed the fit using the parameters for each of the idealized environments and found that the warm ionized medium model resulted in the best fit to the data.

The spinning dust curve for the warm ionized medium fits the data extremely well, and from the fit we found that the column density required to produce the observed spinning dust emission is $N_{\mathrm{H}}^{\mathrm{SD}}=(3.8 \pm 0.5) \times 10^{22} \mathrm{H} \mathrm{cm}^{-2}$, which is consistent with the range of values of the total column density obtained from the DustEm analysis (Section 3.2). Another method of confirming the strength of the AME is to compare to AME observed in other environments. To do this, the $\sim 30 \mathrm{GHz}$ flux density is generally normalized by the $100 \mu \mathrm{m}$ flux density. This quantity, often called the dust emissivity, was computed for RCW175 and we found a value of $(5.8 \pm 1.1) \times 10^{-4}$, which is consistent with the value computed by Davies et al. (2006) for the diffuse dust at intermediate latitudes. These two results imply that the parameters we obtain for the AME in RCW175 are both physical and meaningful.

To investigate the origin of the AME component observed in Figure 12 we adopt the CBI $31 \mathrm{GHz}$ map as a tracer of the AME. This is a reasonable assumption given that the free-free and thermal dust components alone only predict a $31 \mathrm{GHz}$ flux density of $1.7 \mathrm{Jy}$ compared to the measured value of $6.0 \pm$ $0.3 \mathrm{Jy}$. This is an excess of $4.3 \mathrm{Jy}$ implying that $\approx 70 \%$ of the $\mathrm{CBI}$ emission is due to $\operatorname{AME}(14.3 \sigma)$. We note that this is a larger fraction of AME than was found by Dickinson et al. (2009) as they fixed the spectral index of the low-frequency emission at a value of -0.12 . However, even if we fit a power law component with a fixed spectral index of -0.12 , we find that the fraction of $\mathrm{AME}$ is still $\approx 60 \%(12.7 \sigma)$.

Since we know that there is the possibility of ongoing star formation in RCW175, we investigated the correlation between the AME and the location of the YSO candidates identified in Section 3.5. Massive YSOs form in ultracompact H II (UCH II) regions, which have densities $\gtrsim 10^{4} \mathrm{H} \mathrm{cm}^{-3}$ and hence are optically thick. Therefore, the free-free emission originating from the ionized gas is self-absorbed, which results in a turnover in the spectrum from $\alpha=-0.12$ to $\alpha \approx 2$. The exact frequency of this turnover depends on the density of the UCH II region, but can occur up to tens of GHz. Of the six YSO candidates identified within the vicinity of RCW175, only one (source 40) was located in G29.0-0.6 and this is situated $\approx 1$ arcmin from the peak of the AME. The pointing accuracy of the CBI is $\approx 0.5$ arcmin (Taylor et al. 2011), hence making it unlikely that the source of the excess emission in G29.0-0.6 is due to an UCH II region. Similarly, none of the six YSO candidates are associated with the peak of the AME in G29.1-0.7, again suggesting that the AME in this region is not due to an UCH II region.

Having ruled out the possibility of the AME in RCW175 being due to self-absorbed free-free emission, we next want to investigate the connection between the AME and the dust properties. Therefore, we compare the CBI $31 \mathrm{GHz}$ map with the dust parameter maps discussed in Section 3.2 to identify which parameters, if any, correlate well with the AME. In Figure 8, we display the dust parameter maps with the contours of the $31 \mathrm{GHz}$ emission overlaid. It is apparent that the $31 \mathrm{GHz}$ emission is not strongly spatially correlated with the PAH abundance. The deficit of PAHs within the shell of G29.1-0.7 is coincident with the AME observed in this component, while there appears to be a small collection of PAHs present toward the AME originating from G29.0-0.6.

Looking at the VSG abundances relative to the $31 \mathrm{GHz}$ emission, we find that the VSGs are located in the interior of 
both G29.0-0.6 and G29.1-0.7. We find that only the VSGs in G29.0-0.6 are correlated with the $31 \mathrm{GHz}$ emission. The VSGs present in G29.1-0.7 are centered on the location of the B1 II star at the center of the shell, which is clearly offset from the AME emission. This suggests that the AME is not being produced by the VSGs, and is not directly associated with the source of ionization.

Although the hydrogen column density is increased toward G29.0-0.6, and decreased toward G29.1-0.7, there is no apparent correlation with the AME. However, the maps of the ERF strength and $T_{\text {dust }}$ appear to be very well correlated with the CBI emission. Not only is the correlation present toward G29.0-0.6, but it is also present toward G29.1-0.7. As stated previously, within G29.1-0.7, the ERF is enhanced toward the dust filament along the edge of the shell, implying that the ionization front is not uniform in all directions. The correlation between the $\mathrm{AME}$ and the ERF and the warmer dust implies that the AME is dependent on the strength of the ERF, and suggests that it is the excitation of the ERF that is producing the AME. This result is consistent with the result found in the Perseus molecular cloud, where the strength of the ERF appeared to be enhanced in regions of AME (Tibbs et al. 2011), which therefore could be the result of electric dipole emission from spinning dust grains being spun up by photon-grain interactions. Such photon-grain interactions may also cause the grains to become dehydrogenated and, as discussed by Draine \& Lazarian (1998), this will have a strong effect on the electric dipole moment of the grain, which will also affect the spinning dust emissivity. The observed correlation between the AME and the ERF in both RCW175 and the Perseus molecular cloud is in agreement with the spinning dust theory, where, if all other parameters are fixed, the spinning dust emissivity increases with increasing ERF strength (e.g., Ali-Haïmoud et al. 2009; Ysard et al. 2011).

We also note that it is possible that a fraction of the excess emission could be due to magnetic dipole emission produced by thermal fluctuations in the magnetism of the grains (Draine \& Lazarian 1999). According to the theory, the magnitude of the magnetic fluctuations depends on the magnetic properties of the grain material, and any strongly magnetic grain material will almost certainly contain $\mathrm{Fe}$. Indeed, recent results from Peimbert \& Peimbert (2010) has shown that in H II regions, at least $\sim 40 \%$ of the Fe atoms are embedded in dust grains, with this fraction increasing to $\sim 97 \%$ in high metallicity, high density $\mathrm{H}$ II regions. It is difficult to quantify the exact contribution originating from magnetic dipole emission, but one possible method to help distinguish between the two emission mechanisms would be to observe the polarization of the AME. Electric dipole emission is predicted to be only slightly polarized (a fractional polarization of $<1 \%$ above $30 \mathrm{GHz}$; Lazarian \& Draine 2000), while magnetic dipole emission from single domain magnetic grains is expected to be highly polarized (a fractional polarization of up to $\sim 40 \%$; Draine \& Lazarian 1999). No such observations of RCW175 have yet been performed, however, recent observations by Harvey-Smith et al. (2011) have shown that the magnetic field strength toward Galactic $\mathrm{H}$ II regions is similar to the typical magnetic field strength of the diffuse ISM, implying that there is very little polarized emission in these environments. This leads us to believe that the contribution of magnetic dipole emission from single domain magnetic grains in RCW175 is minimal. However, we note that magnetic dipole emission arising from grains containing magnetic inclusions is expected to be relatively unpolarized (a fractional polarization of $<1 \%$; Draine \& Lazarian 1999), and as such, we cannot completely rule out a contribution from magnetic dipole emission.

It is interesting that the AME in G29.1-0.7 is not spatially correlated with the surrounding PDR, where the PAHs are concentrated, but is actually originating from a region just on the interior of the PDR. As we have shown in Figure 8, this region has an enhanced ERF compared to the rest of G29.1-0.7. Recent work by Bernard-Salas et al. (2012) has shown that in the Orion Bar PDR, there is a clear stratification present regarding the major gas ions. They find that although the $[\mathrm{CII}]$ line at $158 \mu \mathrm{m}$ traces the dust in the PDR quite well, the [N II] line at $122 \mu \mathrm{m}$ is not spatially correlated with the dust, but actually originates closer to the ionizing source. This is interesting as we appear to be finding a similar result with the AME in G29.1-0.7 not originating from the PDR, but rather originating closer to the ionization source. In fact, Hoang et al. (2010) investigated the effect of impulsive excitation due to individual ion-grain collisions, and found that these impulses result in a broadening of the spinning dust spectrum to higher frequencies and an increase in the spinning dust emissivity. Furthermore, a recent analysis by Ysard et al. (2011) has shown that the spinning dust model is sensitive to the abundance of the major gas ions, with both the peak frequency and the spinning dust emissivity increasing with ion abundance. This may be what we are observing here, explaining why the AME is not coincident with the PDR but is originating from a region closer to the source of ionization due to the stratification of the gas ions in the PDR. Further information regarding the distribution of the major gas ions within RCW175 is required to test this hypothesis but, to date, no such observations exist.

\section{SUMMARY}

In this work, we have performed a detailed analysis of the environment of RCW175. For this purpose, we used photometric data from $21 \mathrm{~cm}(1.4 \mathrm{GHz})$ to $8 \mu \mathrm{m}(37.5 \mathrm{THz})$ in conjunction with $\mathrm{HI}, \mathrm{CO}$, and RRL spectral data. By looking at the photometric data, we found that RCW175 is not a single H II region, but consists, in fact, of two separate H II regions, G29.10.7 and G29.0-0.6. G29.1-0.7 is larger, more evolved, and most likely the older of the two regions; while G29.0-0.6 is more compact, more dusty, and likely younger. G29.1-0.7 contains the ionizing B1 II star, S65-4, at its center, while G29.0-0.6 is characterized by the presence of filamentary pillars. Both components are clearly associated with each other, given the small spread in velocities, and we suggest that G29.0-0.6 may have been produced as a consequence of triggering from the expansion of G29.1-0.7 into the surrounding ISM.

Using the dust model DusTEM, we characterized the dust properties within the region and we found that there is a significant depletion of PAHs within the larger, diffuse shell of G29.1-0.7, and to a lesser extent within G29.0-0.6. We also found that the abundance of the VSGs is increased toward the interior of both G29.1-0.7 and G29.0-0.6. Both these results are consistent with current theories on the formation of $\mathrm{H}$ II regions, with the PAHs concentrated in the surrounding PDRs, while the warmer dust originates from within the center of the shell. Recent work has hinted that the $24 \mu \mathrm{m}$ emission originating from within the HiI region might not be due to VSGs, but to a population of very warm BGs, emitting thermally at $24 \mu \mathrm{m}$ (e.g., Everett \& Churchwell 2010; Paladini et al. 2012; Salgado et al. 2012).

Looking at the available spectral data we hypothesized that the ionized gas has become dissociated from the molecular gas, 
and we computed a distance of $3.2 \pm 0.2 \mathrm{kpc}$ to RCW175 and a corresponding Galactocentric distance of $5.4 \pm 0.3 \mathrm{kpc}$. We used the $24 \mu \mathrm{m}$ emission to compute an estimate of the SFR for the region of $(12.6 \pm 1.9) \times 10^{-5} M_{\odot} \mathrm{yr}^{-1}$, and also identified six YSO candidates in its vicinity. Additionally, we calculated that the total mass of dust in RCW175 is $215 \pm 53 M_{\odot}$.

Finally, we produced a complete SED from the radio to the mid-IR for the entire complex, and found a clear excess of emission at centimeter wavelengths. This excess was well fitted with a spinning dust model using typical parameters for the warm ionized medium. By comparing the AME with the parameter maps produced by the dust modeling analysis, we found that the AME is not spatially correlated with either the abundance of the PAHs or VSGs, but is much more correlated with the strength of the ERF and hence the dust temperature. This is similar to the result observed for the AME in the Perseus molecular cloud by Tibbs et al. (2011), and hence we suggest that the ERF is playing an important role in causing the dust grains to spin. Further investigation of the AME with respect to the location of the YSO candidates rules out the possibility of the AME originating from an $\mathrm{UCH}$ II region, and based on the fact that the AME in G29.1-0.7 originates from the interior of the PDR, we hypothesize that the AME is due to spinning dust, but that there is a significant contribution to the spinning dust model from the major gas ions.

Further studies like the current one need to be performed in order to fully understand if this connection between the AME and the ERF is found throughout the Galaxy or only occurs in certain environments, and further modeling will help us understand the role of the gas ions in the spinning dust models.

We thank the anonymous referee for providing useful comments that improved the content of this paper. We also thank Mark Calabretta and Lister Staveley-Smith for help with the RRL data. This work has been performed within the framework of a NASA/ADP ROSES-2009 grant No. 09-ADP090059. C.D. acknowledges an STFC Advanced Fellowship and EU Marie Curie IRG grant under the FP7. This work is based in part on observations made with the Spitzer Space Telescope, which is operated by the Jet Propulsion Laboratory, California Institute of Technology under a contract with NASA. This publication makes use of molecular line data from the Boston University-FCRAO Galactic Ring Survey (GRS). The GRS is a joint project of Boston University and Five College Radio Astronomy Observatory, funded by the National Science Foundation under grants AST-9800334, AST-0098562, AST-0100793, AST-0228993, and AST-0507657. The National Radio Astronomy Observatory is a facility of the National Science Foundation operated under cooperative agreement by Associated Universities, Inc. PACS has been developed by a consortium of institutes led by MPE (Germany) and including UVIE (Austria); KU Leuven, CSL, IMEC (Belgium); CEA, LAM (France); MPIA (Germany); INAF-IFSI/OAA/OAP/OAT, LENS, SISSA (Italy); IAC (Spain). This development has been supported by the funding agencies BMVIT (Austria), ESA-PRODEX (Belgium), CEA/CNES (France), DLR (Germany), ASI/INAF (Italy), and CICYT/MCYT (Spain). SPIRE has been developed by a consortium of institutes led by Cardiff University (UK) and including Univ. Lethbridge (Canada); NAOC (China); CEA, LAM (France); IFSI, Univ. Padua (Italy); IAC (Spain); Stockholm Observatory (Sweden); Imperial College London, RAL, UCLMSSL, UKATC, Univ. Sussex (UK); and Caltech, JPL, NHSC, Univ. Colorado (USA). This development has been supported by national funding agencies: CSA (Canada); NAOC (China); CEA, CNES, CNRS (France); ASI (Italy); MCINN (Spain); SNSB (Sweden); STFC (UK); and NASA (USA).

\section{REFERENCES}

Ali-Haïmoud, Y., Hirata, C. M., \& Dickinson, C. 2009, MNRAS, 395, 1055 Alonso-Herrero, A., Rieke, G. H., Rieke, M. J., et al. 2006, ApJ, 650, 835 Alves, M. I. R., Davies, R. D., Dickinson, C., et al. 2012, MNRAS, 422, 2429 AMI Consortium: Scaife, A. M. M., Hurley-Walker, N., et al. 2009, MNRAS, 394, L46

Anderson, L. D., \& Bania, T. M. 2009, ApJ, 690, 706

Bernard, J.-P., Paradis, D., Marshall, D. J., et al. 2010, A\&A, 518, L88

Bernard-Salas, J., Habart, E., Arab, H., et al. 2012, A\&A, 538, A37

Blitz, L., Fich, M., \& Stark, A. A. 1982, ApJS, 49, 183

Burton, W. B., \& Gordon, M. A. 1978, A\&A, 63, 7

Calzetti, D., Kennicutt, R. C., Engelbracht, C. W., et al. 2007, ApJ, 666, 870

Calzetti, D., Wu, S.-Y., Hong, S., et al. 2010, ApJ, 714, 1256

Carey, S. J., Noriega-Crespo, A., Mizuno, D. R., et al. 2009, PASP, 121, 76 Casassus, S., Dickinson, C., Cleary, K., et al. 2008, MNRAS, 391, 1075

Castellanos, P., Casassus, S., Dickinson, C., et al. 2011, MNRAS, 411, 1137 Chomiuk, L., \& Povich, M. S. 2011, AJ, 142, 197

Churchwell, E., Babler, B. L., Meade, M. R., et al. 2009, PASP, 121, 213 Compiègne, M., Flagey, N., Noriega-Crespo, A., et al. 2010, ApJ, 724, L44 Compiègne, M., Verstraete, L., Jones, A., et al. 2011, A\&A, 525, A103 Condon, J. J., Cotton, W. D., Greisen, E. W., et al. 1998, AJ, 115, 1693 Crampton, D., Georgelin, Y. M., \& Georgelin, Y. P. 1978, A\&A, 66, 1 Davies, R. D., Dickinson, C., Banday, A. J., et al. 2006, MNRAS, 370, 1125 Deharveng, L., Schuller, F., Anderson, L. D., et al. 2010, A\&A, 523, A6 Deharveng, L., Zavagno, A., \& Caplan, J. 2005, A\&A, 433, 565 Desert, F. X., Boulanger, F., \& Shore, S. N. 1986, A\&A, 160, 295 Dickinson, C., Casassus, S., Davies, R. D., et al. 2010, MNRAS, 407, 2223 Dickinson, C., Davies, R. D., Allison, J. R., et al. 2009, ApJ, 690, 1585 Dickinson, C., Davies, R. D., Bronfman, L., et al. 2007, MNRAS, 379, 297 Dickinson, C., Davies, R. D., \& Davis, R. J. 2003, MNRAS, 341, 369

Draine, B. T. 2011, ApJ, 732, 100

Draine, B. T., \& Lazarian, A. 1998, ApJ, 508, 157

Draine, B. T., \& Lazarian, A. 1999, ApJ, 512, 740

Elmegreen, B. G. 1998, in ASP Conf. Ser. 148, Origins, ed. C. E. Woodward,

J. M. Shull, \& H. A. Thornson, Jr. (San Francisco, CA: ASP), 150

Everett, J. E., \& Churchwell, E. 2010, ApJ, 713, 592

Fich, M., Blitz, L., \& Stark, A. A. 1989, ApJ, 342, 272

Fitzgerald, M. P. 1970, A\&A, 4, 234

Flagey, N., Boulanger, F., Noriega-Crespo, A., et al. 2011, A\&A, 531, A51

Forbes, D. 1989, A\&AS, 77, 439

Génova-Santos, R., Rebolo, R., Rubiño-Martín, J. A., López-Caraballo, C. H., \& Hildebrandt, S. R. 2011, ApJ, 743, 67

Georgelin, Y. M., \& Georgelin, Y. P. 1976, A\&A, 49, 57

Green, D. A. 2009, Bull. Astron. Soc. India, 37, 45

Griffin, M. J., Abergel, A., Abreu, A., et al. 2010, A\&A, 518, L3

Handa, T., Sofue, Y., Nakai, N., Hirabayashi, H., \& Inoue, M. 1987, PASJ, 39, 709

Harvey-Smith, L., Madsen, G. J., \& Gaensler, B. M. 2011, ApJ, 736, 83

Haynes, R. F., Caswell, J. L., \& Simons, L. W. J. 1978, Aust. J. Phys. Astrophys. Suppl., 45, 1

Helfand, D. J., Becker, R. H., White, R. L., Fallon, A., \& Tuttle, S. 2006, AJ, 131,2525

Hoang, T., Draine, B. T., \& Lazarian, A. 2010, ApJ, 715, 1462

Hoang, T., Lazarian, A., \& Draine, B. T. 2011, ApJ, 741, 87

Hollenbach, D. J., \& Tielens, A. G. G. M. 1997, ARA\&A, 35, 179

Inoue, A. K. 2002, ApJ, 570, 688

Jackson, J. M., Rathborne, J. M., Shah, R. Y., et al. 2006, ApJS, 163, 145

Jarosik, N., Bennett, C. L., Dunkley, J., et al. 2011, ApJS, 192, 14

Langston, G., Minter, A., D’Addario, L., et al. 2000, AJ, 119, 2801

Lazarian, A., \& Draine, B. T. 2000, ApJ, 536, L15

Li, A., \& Draine, B. T. 2001, ApJ, 554, 778

Lockman, F. J. 1989, ApJS, 71, 469

Markwardt, C. B. 2009, in ASP Conf. Ser. 411, Astronomical Data Analysis Software and Systems XVIII, ed. D. A. Bohlender, D. Durand, \& P. Dowler (San Francisco, CA: ASP), 251

Mathis, J. S., Mezger, P. G., \& Panagia, N. 1983, A\&A, 128, 212

Mezger, P. G., \& Henderson, A. P. 1967, ApJ, 147, 471

Mezger, P. G., Smith, L. F., \& Churchwell, E. 1974, A\&A, 32, 269

Miville-Deschênes, M.-A., \& Lagache, G. 2005, ApJS, 157, 302

Molinari, S., Swinyard, B., Bally, J., et al. 2010, PASP, 122, 314

Paladini, R., Davies, R. D., \& De Zotti, G. 2004, MNRAS, 347, 237 
Paladini, R., Umana, G., Veneziani, M., et al. 2012, ApJ, submitted Peimbert, A., \& Peimbert, M. 2010, ApJ, 724, 791

Pérez-González, P. G., Kennicutt, R. C., Jr., Gordon, K. D., et al. 2006, ApJ, 648, 987

Pilbratt, G. L., Riedinger, J. R., Passvogel, T., et al. 2010, A\&A, 518, L1

Planck Collaboration, Ade, P. A. R., Aghanim, N., et al. 2011, A\&A, 536, A20

Poglitsch, A., Waelkens, C., Geis, N., et al. 2010, A\&A, 518, L2

Povich, M. S., Stone, J. M., Churchwell, E., et al. 2007, ApJ, 660, 346

Rebull, L. M., Padgett, D. L., McCabe, C.-E., et al. 2010, ApJS, 186, 259

Reed, B. C. 2003, AJ, 125, 2531

Reich, P., \& Reich, W. 1986, A\&AS, 63, 205

Reich, W. 1982, A\&AS, 48, 219

Reich, W., Fuerst, E., Reich, P., \& Reif, K. 1990a, A\&AS, 85, 633

Reich, W., Reich, P., \& Fuerst, E. 1990b, A\&AS, 83, 539

Reif, K., Reich, W., Steffen, P., Müller, P., \& Weiland, H. 1987, Mitt. Astron. Ges. Hamburg, 70, 419

Relaño, M., Lisenfeld, U., Pérez-González, P. G., Vílchez, J. M., \& Battaner, E. 2007, ApJ, 667, L141

Robitaille, T. P., Meade, M. R., Babler, B. L., et al. 2008, AJ, 136, 2413
Rodgers, A. W., Campbell, C. T., \& Whiteoak, J. B. 1960, MNRAS, 121, 103 Salgado, F., Berne, O., Adams, J. D., et al. 2012, ApJ, 749, L21

Sanders, D. B., Clemens, D. P., Scoville, N. Z., \& Solomon, P. M. 1986, ApJS, 60,1

Sharpless, S. 1959, ApJS, 4, 257

Silsbee, K., Ali-Haïmoud, Y., \& Hirata, C. M. 2011, MNRAS, 411, 2750

Smith, L. F., Biermann, P., \& Mezger, P. G. 1978, A\&A, 66, 65

Staveley-Smith, L., Juraszek, S., Koribalski, B. S., et al. 1998, AJ, 116, 2717

Stil, J. M., Taylor, A. R., Dickey, J. M., et al. 2006, AJ, 132, 1158

Taylor, A. C., Jones, M. E., Allison, J. R., et al. 2011, MNRAS, 418, 2720

Tibbs, C. T., Flagey, N., Paladini, R., et al. 2011, MNRAS, 418, 1889

Tibbs, C. T., Watson, R. A., Dickinson, C., et al. 2010, MNRAS, 402, 1969

Todorović, M., Davies, R. D., Dickinson, C., et al. 2010, MNRAS, 406, 1629

Traficante, A., Calzoletti, L., Veneziani, M., et al. 2011, MNRAS, 416, 2932

Turner, D. G. 1980, ApJ, 240, 137

Vidal, M., Casassus, S., Dickinson, C., et al. 2011, MNRAS, 414, 2424

Watson, R. A., Rebolo, R., Rubiño-Martín, J. A., et al. 2005, ApJ, 624, L89

Ysard, N., Juvela, M., \& Verstraete, L. 2011, A\&A, 535, A89

Ysard, N., \& Verstraete, L. 2010, A\&A, 509, A12

Zavagno, A., Deharveng, L., Comerón, F., et al. 2006, A\&A, 446, 171 